\title{
Ultrashort-Pulse Laser Machining
}

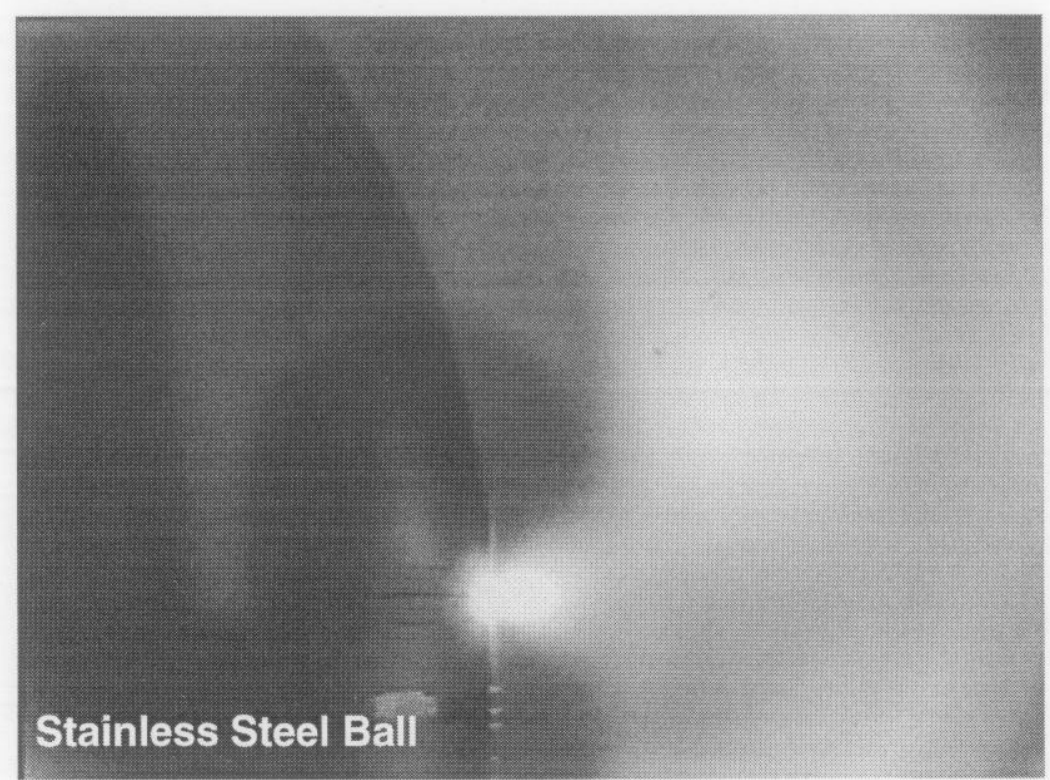

September 1998 


\section{DISCLAIMER}

This document was prepared as an account of work sponsored by an agency of the United States Government. Neither the United States Government nor the University of California nor any of their employees, makes any warranty, express or implied, or assumes any legal liability or responsibility for the accuracy, completeness, or usefulness of any information, apparatus, product, or process disclosed, or represents that its use would not infringe privately owned rights. Reference herein to any specific commercial product, process, or service by trade name, trademark, manufacturer, or otherwise, does not necessarily constitute or imply its endorsement, recommendation, or favoring by the United States Government or the University of California. The views and opinions of authors expressed herein do not necessarily state or reflect those of the United States Government or the University of California, and shall not be used for advertising or product endorsement purposes.

This report has been reproduced directly from the best available copy.

Available to DOE and DOE contractors from the Office of Scientific and Technical Information

P.O. Box 62, Oak Ridge, TN 37831

Prices available from (615) 576-8401, FTS 626-8401

Available to the public from the

National Technical Information Service

U.S. Department of Commerce

5285 Port Royal Rd.,

Springfield, VA 22161

Work performed under the auspices of the U.S. Department of Energy by Lawrence Livermore National Laboratory under Contract W-7405-ENG-48. 


\section{Ultrashort-Pulse Laser Machining}

\section{M.D. Perry, B.C. Stuart, P.S. Banks, H.T. Nguyen, M.D. Feit, A.M. Rubenchik, and J.A. Sefcik}

September 1998

Cover photo: Femtosecond laser system machining stainless steel. Femtosecond lasers cut without melting collateral damage. 


\section{Contents}

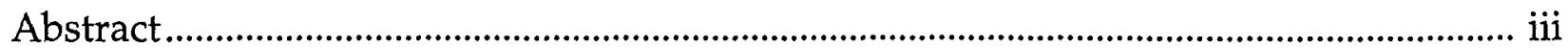

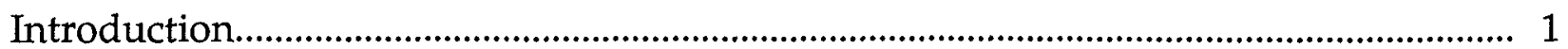

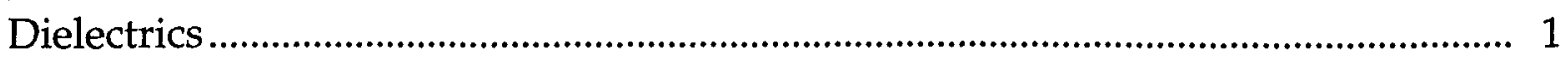

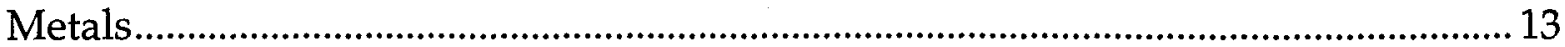

I

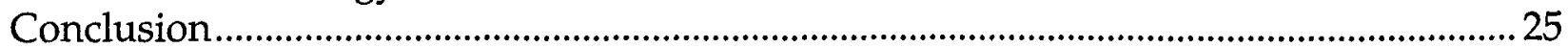

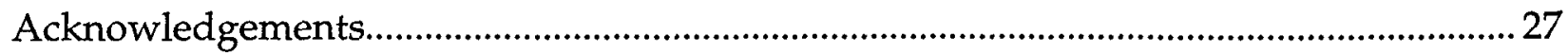

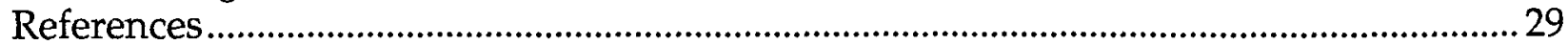




\section{Figures}

Figure 1. (a) Drilling of enamel (tooth) with conventional $1053 \mathrm{~nm}$, nanosecond pulses (ablation threshold $=30 \mathrm{~J} / \mathrm{cm}^{2}$ for $\mathrm{p}=2 \mathrm{nsec}$ )

Figure 2. Observed values of damage threshold at $1053 \mathrm{~nm}$ for fused silica $(\bullet)$ and CaF2 $(\bullet)$.

Figure 3. Schematic of collisional ionization with the approximation that the kinetic energy in the final state is shared equally by the electrons ...

Figure 4. Calculated evolution of free electron density for a 100-fs, 1053-nm pulse (dashed curve) of peak irradiance $=10 \mathrm{TW} / \mathrm{cm}^{2}$ in fused silica

Figure 5. Reflectivity of fused silica surface as a function of laser irradiance for $\lambda=1053 \mathrm{~nm}$ and $\tau_{\mathrm{p}}=350 \mathrm{fs}$

Figure 6. Measured and calculated damage thresholds of fused silica in the infrared and visible $(526 \mathrm{~nm})$

Figure 7. Temperature increase of bulk tooth due to drilling with 1.4-nanosecond (circles) and 350-femtosecond (squares) laser pulses

Figure 8. Cuts in explosive pellet (PETN) by a Ti:sapphire laser operating at $120 \mathrm{fs}(\mathrm{a})$ and $0.6 \mathrm{~ns} \mathrm{(b)}$

Figure 9. Cuts in stainless steel performed with $1054 \mathrm{~nm}$ laser pulses with a duration of (a) $1.4 \mathrm{~ns}$ at a fluence of $52 \mathrm{~J} / \mathrm{cm}^{2}$ and (b) $350 \mathrm{fs}$ and $14 \mathrm{~J} / \mathrm{cm}^{2}$

Figure 10. Calculated temperature profiles in gold irradiated at $1054 \mathrm{~nm}$ and a fluence of $0.5 \mathrm{~J} / \mathrm{cm}^{2}$ for 1,10 , and 100 ps pulses

Figure 11. Damage threshold of gold at $1054 \mathrm{~nm}$ as a function of pulse duration [from ref. 29]

Figure 12. Calculated temperature (a) and pressure (b) of aluminum irradiated by $1053-\mathrm{nm}, 350-\mathrm{fs}$ laser pulses at a fluence of $5 \mathrm{~J} / \mathrm{cm}^{2}$ 18

Figure 13. Calculated temperature (a) and pressure (b) of aluminum following irradiation by $1053-\mathrm{nm}, 350-\mathrm{fs}$ laser pulses at a fluence of $5 \mathrm{~J} / \mathrm{cm}^{2}$

Figure 14. Calculated temperature (a) and pressure (b) of aluminum following irradiation by a 1053-nm, 1-ns laser pulse at a fluence of $20 \mathrm{~J} / \mathrm{cm}^{2}$ (same depth of material removed as in Figure 13)

Figure 15. Depth of material removed per laser pulse for stainless steel and $\lambda=825 \mathrm{~nm}$

Figure 16. Holes drilled through $1 \mathrm{~mm}$ stainless steel with 120 -fs laser pulses at $1 \mathrm{kHz}$ at $45^{\circ}$ 23

Figure 17. Holes drilled through $1 \mathrm{~mm}$ stainless steel for fuel injector nozzles .............24

Figure 18. Photograph of a 15-W average power femtosecond laser machining system developed for industrial operation 


\begin{abstract}
A new type of material processing is enabled with ultrashort $(t<10 \mathrm{ps})$ laser pulses. Cutting, drilling, sculpting of all materials (biologic materials, ceramics, sapphire, silicon carbide, diamond, metals) occurs by new mechanisms that eliminate thermal shock or collateral damage. High-precision machining to submicron tolerances is enabled resulting in high surface quality and negligible heat affected zone.
\end{abstract}




\section{Introduction}

Conventional mechanical lathes and machine tools are effective for machining simplified shapes (cutting, drilling) in metals down to approximately $200-\mu \mathrm{m}(8 \mathrm{mil})$ kerf width at depths of approximately $1 \mathrm{~mm}$ (aspect ratio <5:1). For finer features or irregular shapes, electron beam, electron discharge (EDM) or conventional laser tools are typically used. Conventional laser tools such as those based on $\mathrm{CO}_{2}, \mathrm{Nd}: \mathrm{YAG}$, or copper vapor lasers machine materials by localized heating. Both EDM and existing industrial laser technology heat the material to the melting or boiling point resulting in thermal stress to the remaining material and often a heat affected zone. Higher precision or higher aspect ratio (e.g., $100-\mu \mathrm{m}$ holes thru $1 \mathrm{~mm}$ steel) is difficult to achieve with these techniques. Furthermore, non-metals (e.g., ceramics, SiC, diamond, sapphire, bone, etc) are very difficult to machine using EDM or conventional laser processing. Laser processing by molecular dissociation in organic (and some inorganic) materials can be achieved with ultraviolet lasers (e.g., excimer lasers- $\mathrm{KrF}, \mathrm{XeCl}$ ) but this photodissociation mechanism is not applicable to metals.

By using ultrashort $(\tau<10 \mathrm{ps})$ laser pulses any material can be machined to very high precision. The ability to machine any material-including high bandgap dielectrics such as $\mathrm{SiC}$, diamond, etc.-is a result of the fact that these ultrashort pulses interact by a mechanism that is very different from that of conventional longer pulse lasers. This interaction is independent of the usual linear absorption properties of the material and is applicable to materials which would otherwise be transparent to the laser wavelength. Machining to micron-scale ( $<0.1 \mathrm{mil})$ precision with no collateral damage to the remaining material is achieved by removing material faster than heat can be conducted to the bulk.

In an effort to introduce the concepts and challenges associated with ultrashort-laser machining, wc have divided this article is into three basic sections: machining of dielectrics; machining of metals; and laser technology.

\section{Dielectrics}

By dielectrics, we generally are referring to materials with no free electrons and low thermal/electrical conductivity. Common materials such as fused silica, sapphire, $\mathrm{SiC}$, diamond, $\mathrm{SiN}$, AlTiC, $\mathrm{ZrO}_{2}$, glass, plastic, bone, cornea, heart tissue, etc. would fall under this category. Semiconductors such as silicon and gallium arsenide would generally not be considered dielectrics by this definition. However, these materials behave similar to dielectrics when machining with ultrashort laser pulses.

Attempts to machine dielectrics with lasers followed shortly after laser induced damage was observed in transparent solids. ${ }^{1-5}$ For pulses longer than a few tens of picoseconds, the generally accepted picture of damage to dielectrics involves the heating of seed electrons by the incident radiation and transfer of this energy to the 
lattice. Damage occurs via conventional heat deposition resulting in melting and boiling of the dielectric material. Because the controlling rate is that of thermal conduction through the lattice, this model predicts a $\tau^{1 / 2}$ dependence of the threshold fluence (energy/area) upon pulse duration $\tau,{ }^{6}$ in reasonably good agreement with numerous experiments which have observed a $\tau^{\alpha}$ scaling with $0.4<\alpha<0.5$ in a variety of dielectric materials from 100 ps to microseconds. ${ }^{7}$ With these conventional lasers, material is removed by thermal ablation wherein the material is locally heated to near the boiling point. Since the boiling point of these materials is very high (typically $>1000^{\circ} \mathrm{C}$ ), this ablation mechanism is accompanied by a strong thermal shock to the remaining bulk material. This thermal shock often results in cracking of the remaining material and uncontrolled material removal. These effects can be observed in Figure 1 where the initial stages of hole drilling in a tooth using a conventional Nd:YAG laser are shown. In Figure 1a, linear absorption due to defects produces inhomogeneous energy absorption across the laser beam. Thermal stresses increase to the point where ablation begins first from the point with the least material strength.

It had been postulated that the $\tau^{1 / 2}$ dependence of the laser damage threshold for dielectrics would break down for pulse durations less than a few picoseconds as the probability of multiphoton ionization of the individual atoms within the dielectric became significant. ${ }^{8}$ In order to quantify the transition from a thermal mechanism of damage to one associated with direct ionization, we began a systematic study of the damage threshold in a variety of dielectric materials in 1993. In all dielectrics which we examined, a strong deviation from the $\tau^{1 / 2}$ dependence of the damage threshold was observed. This deviation occurred in a range from $10 \mathrm{ps}\left(\mathrm{SiO}_{2}\right)$ to $\approx 20 \mathrm{ps}\left(\mathrm{CaF}_{2}\right)$ as shown in Figure 2.9

In all cases, the deviation was accompanied by a dramatic change in morphology of the damaged surface (Figure 1b). With femtosecond pulses, all regions throughout the laser beam profile with sufficient intensity for multiphoton ionization will be removed resulting in extremely fine control of the position of material removal. In addition, the morphology of the surface drilled with femtosecond pulses is characteristic of the internal enamel and dentin. There is no evidence of heat transfer into the surrounding material and no thermal shock cracking in any of the surrounding material (Figure 1c).

Both the change in surface morphology and the deviation from the $\tau^{1 / 2}$ dependence of the damage threshold on pulse duration are predicted by a rapid ionization mechanism. ${ }^{9}$ We will consider fused silica in greater detail in order to describe the model with a specific material. Damage occurs at a threshold of $\approx 2 \mathrm{~J} / \mathrm{cm}^{2}$ for a 1 -ps pulse (Figure 2). With an irradiance of $2 \times 10^{12} \mathrm{~W} / \mathrm{cm}^{2}$, there is no need to invoke some arbitrary number of initial "seed" electrons. ${ }^{1-5,10,11}$ Field-induced multiphoton ionization produces free electrons which are then rapidly accelerated by the laser pulse. For these very short, intense pulses, energy is gained by the newly free electrons from the laser field much faster than it is transferred from the electrons to the lattice. These electrons gain energy from the laser field until they have sufficient energy to 

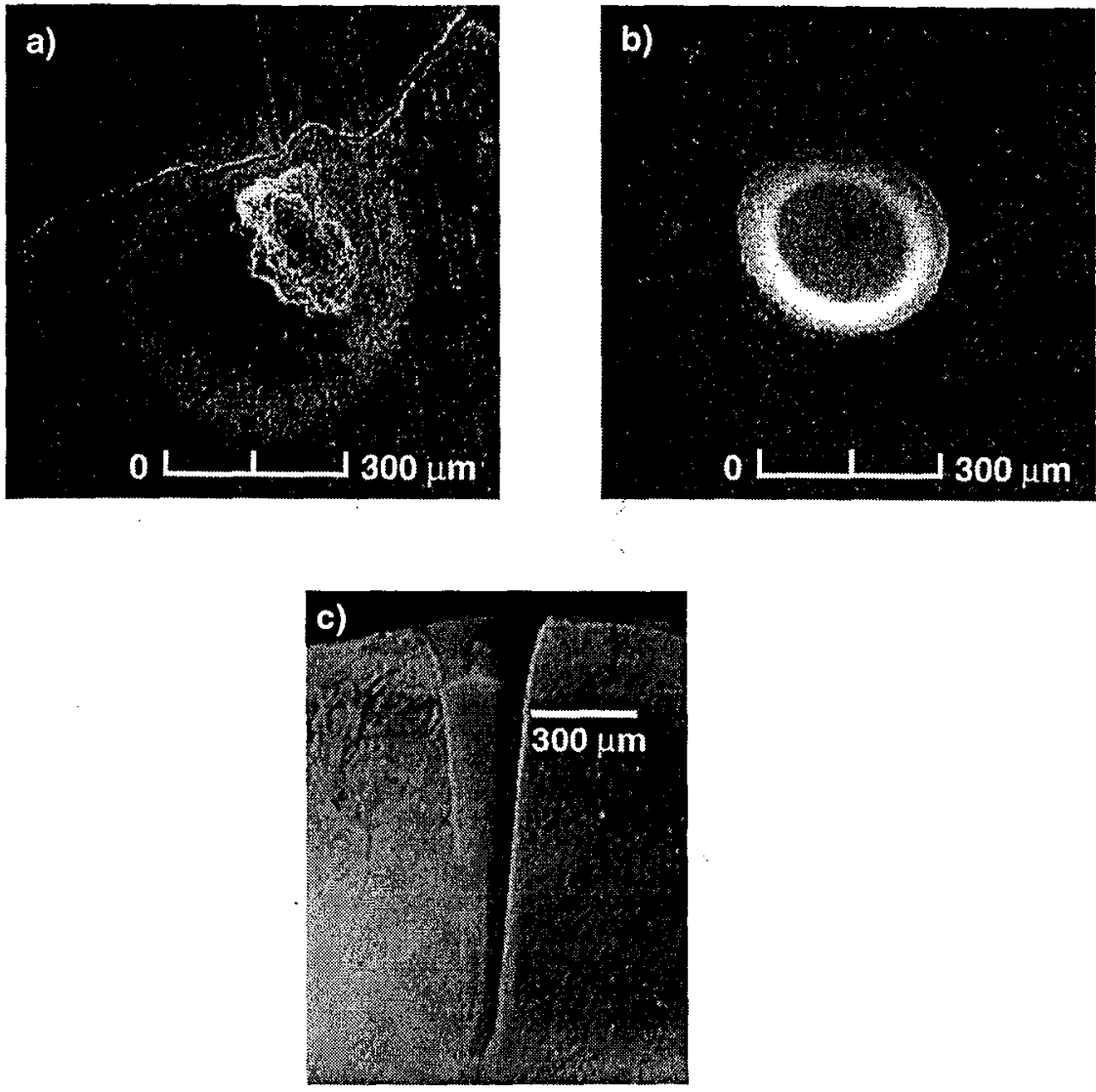

Figure 1. (a) Drilling of enamel (tooth) with conventional $1053 \mathrm{~nm}$, nanosecond pulses (ablation threshold $=30 \mathrm{~J} / \mathrm{cm}^{2}$ for $\mathrm{p}=2 \mathrm{nsec}$ ). (b) Same as in (a) but with the pulse duration reduced to the ultrashort regime (ablation threshold $=3 \mathrm{~J} / \mathrm{cm}^{2}$ or $\mathrm{p}=0.35 \mathrm{ps}$ ). In both cases, the laser spot size was $300 \mathrm{~m}$. (c) cross section of hole made with 350 fs pulses.

collisionally ionize neighboring atoms thereby producing more free electrons. This process continues until a critical density plasma is reached wherein no further energy deposition from the laser occurs. The actual damage occurs after the pulse has passed, when the dense plasma expands away from the surface.

Plasma formation is quantitatively described by the time dependence of the electron energy distribution function. Since the impact ionization rate is energy dependent, the absorption rate of laser energy requires integrating over the electron energy distribution. When the electrons are strongly driven by intense laser pulses, the energy distribution can differ substantially from a Maxwellian. For material having a bandgap energy that is much larger than the single photon energy, $\mathrm{U}_{\mathrm{I}}>\hbar \omega \omega$, the heating and 


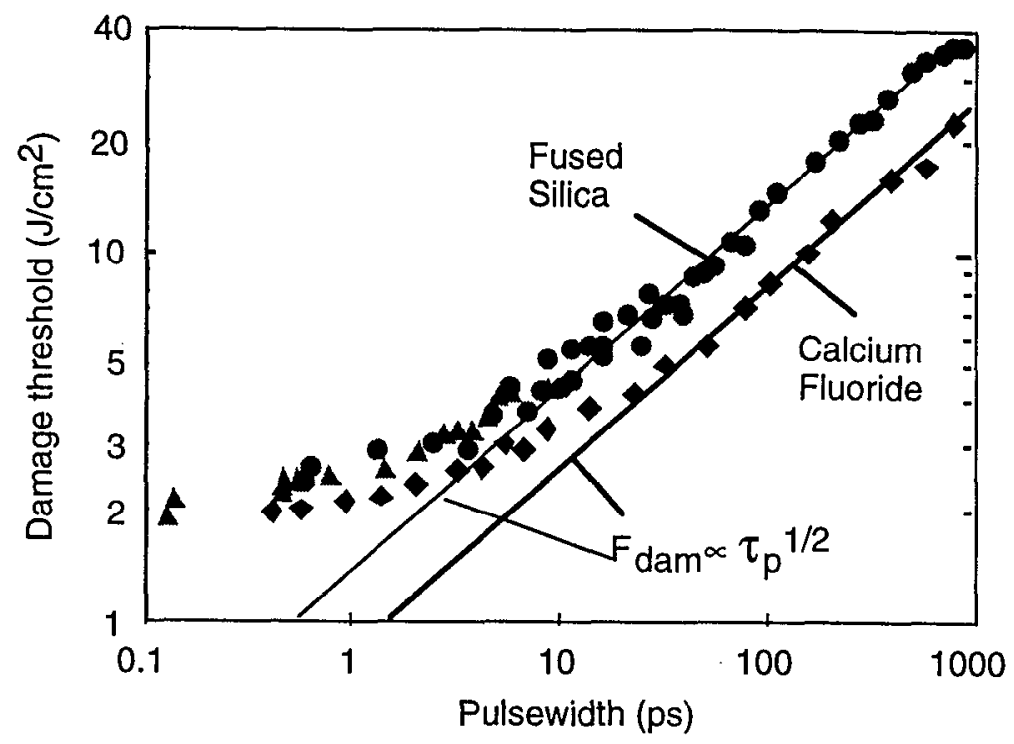

Figure 2. Observed values of damage threshold at $1053 \mathrm{~nm}$ for fused silica ( $\bullet$ ) and CaF2 $(\bullet)$. Solid lines are $1 / 2$ fits to long pulse results, dashed line is fluence required to cause damage in $\mathrm{SiO} 2$ by photoionization alone. Estimated absolute error in data is $\pm 15 \%$.

collisional ionization of conduction electrons can be described by a Fokker-Planck equation 5,12 for the electron distribution function $N(\varepsilon, t)$,

$$
\frac{\partial N(\varepsilon, t)}{\partial t}+\frac{\partial}{\partial \varepsilon}\left[R_{J}(\varepsilon, t) N(\varepsilon, t)-\gamma(\varepsilon) E_{P} N(\varepsilon, t)-D(\varepsilon, t) \frac{\partial N(\varepsilon, t)}{\partial \varepsilon}\right]=S(\varepsilon, t)
$$

where $\varepsilon$ is the electron kinetic energy. The number of electrons with a kinetic energy in the range, $\varepsilon$ to $\varepsilon+d \varepsilon$ at the time, $t$, is given by $N(\varepsilon, t) d \varepsilon$. The square bracket in Eq. 1 represents the change in the electron distribution due to Joule heating, inelastic scattering of phonons, and electron energy diffusion. The Joule heating rate of the electrons is given by,

$$
\mathrm{R}_{\mathrm{J}}(\varepsilon, \mathrm{t})=\frac{\sigma(\varepsilon)}{3} \mathrm{E}_{\mathrm{rms}}(\mathrm{t})^{2}
$$

where $E_{\mathrm{rms}}(t)$ is the electric field of the laser and $\sigma(\varepsilon)=\mathrm{e}^{2} \tau_{\mathrm{m}}(\varepsilon) / \mathrm{m}^{*}\left[1+\omega^{2} \tau_{\mathrm{m}}(\varepsilon)^{2}\right]$ is the ac conductivity of a conduction-band electron of effective mass $m^{*}$. Here, $v_{m}(\varepsilon)=1 / \tau_{m}(\varepsilon)$ is the energy-dependent, electron-phonon transport (momentum) scattering rate. It is instructive to rewrite this expression as

$$
R_{J}(\varepsilon, t)=v_{\text {coll }}(\varepsilon) \frac{e^{2} E(t)^{2}}{4 m \omega^{2}}=v_{\text {coll }}(\varepsilon) U_{p}
$$


where $U_{p}=e^{2} E(t)^{2} / 4 m \omega^{2}=9.33 \times 10^{-14} \mathrm{I}\left(\mathrm{W} / \mathrm{cm}^{2}\right) \lambda^{2}(\mu \mathrm{m})$, is the free electron quiver energy expressed in $\mathrm{eV}$ and $v_{\text {coll }}(\varepsilon)=v_{\mathrm{m}}(\varepsilon)^{*}\left\{\omega^{2} \tau_{\mathrm{m}}(\varepsilon)^{2} /\left[1+\omega^{2} \tau_{\mathrm{m}}(\varepsilon)^{2}\right\} \approx v_{\mathrm{m}}(\varepsilon)\right.$ is the effective electron collision frequency with the lattice. For $\mathrm{SiO}_{2}$, the momentum scattering rate, $v_{\mathrm{m}}(\varepsilon)$, varies between $2 \mathrm{fs}^{-1}$ and $10 \mathrm{fs}^{-1}$ from 1 to $10 \mathrm{eV} \cdot{ }^{13}$ Hence, for a laser wavelength of $1064 \mathrm{~nm}$ and an intensity of $10^{13} \mathrm{~W} / \mathrm{cm}^{2}$, the single electron heating rate is on the order of a few $\mathrm{eV} / \mathrm{fs}$.

The rate of energy transfer from the electrons to the lattice is given by the second term within the brackets, $\gamma(\varepsilon) E_{\mathrm{p}} N(\varepsilon, t)$, where $E_{\mathrm{p}}$ is the energy of a typical phonon and $\gamma(\varepsilon)$ is the rate of electron-phonon energy transfer to the lattice. Note that for pulses on the order of $1 \mathrm{TW} / \mathrm{cm}^{2}$, the electron heating rate, $R_{\mathrm{J}}(\varepsilon, t)$ is much greater than the rate of energy transfer to the lattice, $\gamma(\varepsilon) E_{\mathrm{p}}$. The term $\mathrm{D}(\varepsilon, \mathrm{t}) \partial \mathrm{N} / \partial \varepsilon$ represents electron energy diffusion with a diffusion coefficient given by $D(\varepsilon, t)=2 \varepsilon R_{f}(\varepsilon, t)$.

The final term represents sources and sinks of electrons. Specifically, the total number of electrons created with kinetic energy in the range, $\varepsilon$ to $\varepsilon+d \varepsilon$ at the time, $t$, is given by $\mathrm{S}(\varepsilon, \mathrm{t}) \mathrm{d} \varepsilon$. Free electrons can be created by both multiphoton ionization and collisional (impact) ionization. Electrons that have been accelerated to energies above the ionization potential, $\mathrm{U}_{\mathrm{IP}}$, can collisionally ionize a neighboring atom. An incident electron of kinetic energy, $\varepsilon_{0}=2 \varepsilon+\mathrm{U}_{\mathrm{IP}}$, produces two free electrons of energy, $\varepsilon$, and an ion (hole) of potential energy, $\mathrm{U}_{\mathrm{IP}}$ in the final state (Figure 3). Impact ionization occurs at a rate described by the Keldysh impact formula ${ }^{14}, v_{\mathrm{I}}\left(\varepsilon_{\mathrm{o}}\right)=\chi\left(\varepsilon_{\mathrm{o}} / \mathrm{U}_{\mathrm{IP}}-1\right)^{2}$. For fused silica, the proportionality constant, $\chi=1.5 \times 10^{15} \mathrm{sec}^{-1}$ for incident electrons of energy $<100 \mathrm{eV} .{ }^{13}$

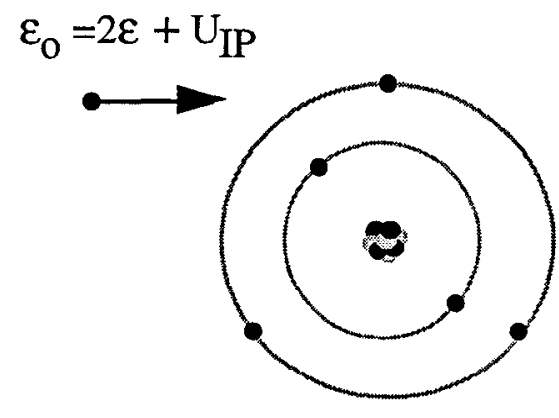

Initial

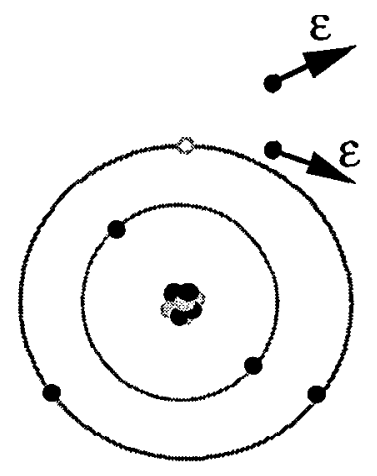

Final

Figure 3. Schematic of collisional ionization with the approximation that the kinetic energy in the final state is shared equally by the electrons. 
The source term for impact ionization can be written as, 12

$$
\begin{aligned}
\mathrm{S}(\varepsilon, \mathrm{t})_{\text {impact }} \mathrm{d} \varepsilon & =2 \mathrm{~N}\left(\varepsilon_{\mathrm{o}}, \mathrm{t}\right) \mathrm{V}_{\mathrm{I}}\left(\varepsilon_{\mathrm{o}}\right) \mathrm{d} \varepsilon_{\mathrm{o}}-\mathrm{N}(\varepsilon, \mathrm{t}) \mathrm{V}_{\mathrm{I}}(\varepsilon) \mathrm{d} \varepsilon \\
& =\left[4 \mathrm{~N}\left(2 \varepsilon+\mathrm{U}_{\mathrm{IP}}, \mathrm{t}\right) \mathrm{V}_{\mathrm{I}}\left(2 \varepsilon+\mathrm{U}_{\mathrm{IP}}\right)-\mathrm{N}(\varepsilon, \mathrm{t}) \mathrm{V}_{\mathrm{I}}(\varepsilon)\right] \mathrm{d} \varepsilon
\end{aligned}
$$

The term, $4 \mathrm{~N}\left(2 \varepsilon+\mathrm{U}_{\mathrm{IP}}, t\right) v_{\mathrm{I}}\left(2 \varepsilon+\mathrm{U}_{\mathrm{IP}}\right)$ represents those electrons born into $\varepsilon$ to $\varepsilon+\mathrm{d} \varepsilon$ by impact ionization, while the term, $\mathrm{N}(\varepsilon, t) v_{\mathrm{I}}(\varepsilon)$ represents those that are lost from this energy bin. The factor of four in the source term can be understood simply from the fact that the two electrons born into $\varepsilon$ to $\varepsilon+d \varepsilon$ from the initial energy, $\varepsilon_{0}=2 \varepsilon+U_{\text {IP }}$ are born into an energy bin, $\mathrm{d} \varepsilon$ which is only half as large as the original (e.g., $\mathrm{d} \varepsilon_{0}=2 \mathrm{~d} \varepsilon$ ).

The total source term is given by,

$$
\begin{aligned}
\mathrm{S}(\varepsilon, \mathrm{t}) \mathrm{d} \varepsilon & =\left[\mathrm{S}(\varepsilon, \mathrm{t})_{\mathrm{PI}}+\mathrm{S}(\varepsilon, \mathrm{t})_{\text {impact }}\right] \mathrm{d} \varepsilon \\
& =\left[\mathrm{S}(\varepsilon, \mathrm{t})_{\mathrm{PI}}+4 \mathrm{~N}\left(2 \varepsilon+\mathrm{U}_{\mathrm{IP}}, \mathrm{t}\right) \mathrm{V}_{\mathrm{I}}\left(2 \varepsilon+\mathrm{U}_{\mathrm{IP}}\right)-\mathrm{N}(\varepsilon, \mathrm{t}) \mathrm{V}_{\mathrm{I}}(\varepsilon)\right] \mathrm{d} \varepsilon
\end{aligned}
$$

The photoionization source term, $S(\varepsilon, t)_{\text {PI }} d \varepsilon$ has been studied extensively in the case of isolated atoms. In the limit that the bandgap of the material is not too much greater than the photon energy, $\mathrm{U}_{\mathrm{IP}} / \mathrm{hv}<4$, and there are no intermediate resonances, simple perturbation theory can be used: $S(\varepsilon, t)_{\mathrm{PI}} \mathrm{d} \varepsilon=\sigma\left(\mathrm{KI} \mathrm{I}^{\mathrm{K}}\right.$ where $\mathrm{K}$ is the number of photons required to ionize the atom, $\sigma^{(\mathrm{K})}$ is the multiphoton ionization cross section, and $\mathrm{I}$ is the photon flux (photons $/ \mathrm{cm}^{2} \mathrm{sec}$ ). The energy of the resulting electron is $\varepsilon=\mathrm{Kh} v-\mathrm{U}_{\mathrm{IP}}$. An example of this case would be fused silica irradiated by $532 \mathrm{~nm}$ light. Four $2.33 \mathrm{eV}$ photons are required to ionize an $\mathrm{SiO}_{2}$ molecule $\left(\mathrm{E}_{\mathrm{b}}=9 \mathrm{eV}\right)$ with a cross section similar to that measured for $\mathrm{NaCl}, \sigma^{(4)}=2 \times 10^{-114} \mathrm{~cm}^{8} / \mathrm{sec}^{3}$. [Ref. 1] If there are intermediate multiphoton resonances involved, more sophisticated expressions for the ionization rate are required. ${ }^{15}$ In the limit that the ionization potential is less than approximately twice the free electron quiver energy, $\mathrm{U}_{\mathrm{IP}}<2 \mathrm{U}_{\mathrm{P}}$, perturbation theory breaks down.

Photoionization is then described by the tunneling theory of Keldysh ${ }^{16}$ or derivatives thereof. 17,18

Direct numerical solution of eq. 1 can be found in ref. 19. However, a very useful approximate solution can be obtained for the total electron density, $N(t)$, in the limit that the electron heating rate is much greater than the rate of energy transfer to the lattice. Note that,

$$
\frac{\mathrm{dN}(\mathrm{t})}{\mathrm{dt}}=\frac{\partial}{\partial \mathrm{t}} \int_{0}^{\infty} \mathrm{N}(\varepsilon, \mathrm{t}) \mathrm{d} \varepsilon
$$


Inserting equations 2-5 into eq. 1 and integrating over energy, we can write the time dependence of the electron density as,

$$
\frac{\mathrm{dN}(\mathrm{t})}{\mathrm{dt}}=\beta(\mathrm{I}) \mathrm{N}(\mathrm{t})+\mathrm{P}(\mathrm{I})
$$

where $\beta=\alpha \mathrm{I}(\mathrm{t})$ contains all the terms associated with electron heating and impact ionization and $\mathrm{P}(\mathrm{I})$ is the photoionization term,

$$
P(I)=\int_{0}^{\infty} S(\varepsilon, t)_{P I} d \varepsilon=N_{0} W(t)
$$

where the term $N_{o}$ is the initial atom density and $W(t)$ is the single atom photoionization rate. Equation 7 is readily solved to yield, .

$$
N(t)=N_{o} \operatorname{Exp}\left[\alpha \int^{t} I\left(t^{\prime}\right) d^{\prime}\right] * \int_{-\infty}^{-t} W\left(t^{\prime}\right) \operatorname{Exp}\left[-\alpha \int^{t^{\prime}} I\left(t^{\prime \prime}\right) d t^{\prime \prime}\right] d t^{\prime}
$$

The avalanche coefficient, $\alpha \approx 0.011 \mathrm{~cm}^{2} \mathrm{ps}^{-1} / \mathrm{GW}$ for fused silica, is found by numerically solving Eq. $1^{9,19}$ or by fitting the experimental data. Note that $1 / \alpha=0.1 \mathrm{~J} / \mathrm{cm}^{2}$ has the units of fluence. Many mode-locked short-pulse lasers exhibit a temporal distribution given by,

$$
I(t)=I_{o} \operatorname{sech}^{2}\left(2 t / \tau_{p}\right)
$$

Inserting this expression into eq. 9 yields,

$$
\mathrm{N}(\mathrm{t})=\mathrm{N}_{\mathrm{o}} \operatorname{Exp}\left[\left(\frac{\alpha \mathrm{I}_{\mathrm{o}} \tau_{\mathrm{p}}}{2}\right) \tanh \left(2 \mathrm{t} / \tau_{\mathrm{p}}\right)\right] * \int_{-\infty}^{-\mathrm{t}} \mathrm{W}\left(\mathrm{t}^{\prime}\right) \operatorname{Exp}\left[-\left(\frac{\alpha \mathrm{I}_{\mathrm{o}} \tau_{\mathrm{p}}}{2}\right) \tanh \left(2 \mathrm{t}^{\prime} / \tau_{\mathrm{p}}\right)\right] \mathrm{dt^{ \prime }}
$$

This is a very useful analytic expression that predicts the time-dependent electron density from both multiphoton and collisional ionization given an expression for the single-atom multiphoton ionization rate, $W(t)$.

Figure 4 shows the evolution of electron density for a $10 \mathrm{TW} / \mathrm{cm}^{2}, 100-\mathrm{fs}$ pulse incident on fused silica. The temporal profile of the laser pulse and the electron density produced by multiphoton ionization alone are included for reference. Because multiphoton ionization is strongly intensity dependent, the electron production takes place principally at the peak of the pulse. For this $100-f s$ duration, multiphoton ionization produces a substantial amount of free electrons. When the electron density produced by multiphoton ionization approaches $\approx 10^{17} \mathrm{~cm}^{-3}$, the collisional ionization rate begins to exceed the multiphoton ionization rate. Once a high free electron density is produced by multiphoton ionization the material no longer has the properties of a 


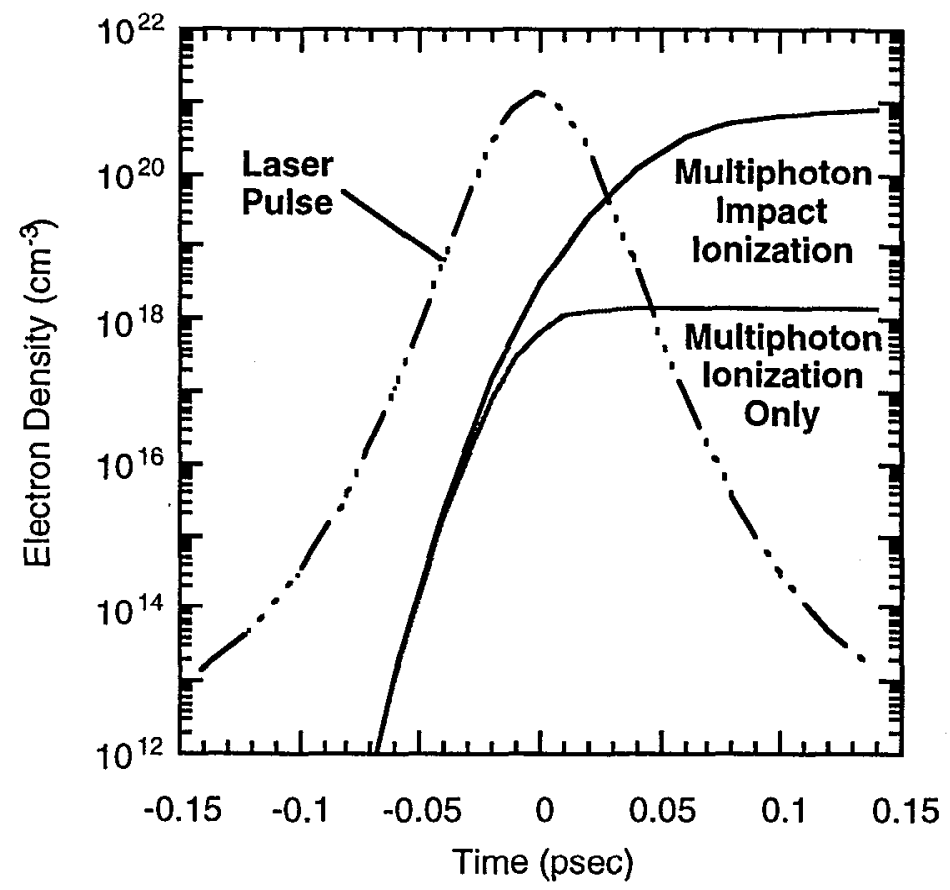

Figure 4. Calculated evolution of free electron density for a 100-fs, 1053-nm pulse (dashed curve) of peak irradiance $=10 \mathrm{TW} / \mathrm{cm}^{2}$ in fused silica. The total electron density including impact ionization and that due to multiphoton ionization alone are shown for comparison.

dielectric. It is now a conductor and will absorb the laser via inverse Bremstrahlung (Joule) heating similar to a metal. It is for this reason that both dielectrics and metals have similar behavior and morphology when machined with ultrashort pulses. In essence, the dielectric is converted to a metallic state within the first few tens of femtoseconds. As the laser intensity decreases past the peak of the pulse, the driving force for both multiphoton and collisional ionization ceases and the free electron density remains constant.

For laser pulses of different duration, the relative fraction of multiphoton ionization to avalanche ionization will change. Multiphoton ionization will contribute a relatively greater fraction of the electron density with shorter pulses while avalanche ionization will dominate with longer pulses. In all cases, the electron density saturates at the critical density, $\mathrm{N}_{\mathrm{c}}$. The critical density is the density at which the plasma frequency, $\omega_{\mathrm{pe}}(\mathrm{rad} / \mathrm{sec})=\left(4 \pi \mathrm{N}_{\mathrm{e}} \mathrm{e}^{2} / \mathrm{m}\right)^{1 / 2}=\left[2.21 \times 10^{8} \mathrm{~N}_{\mathrm{e}}\left(\mathrm{cm}^{-3}\right)\right]^{1 / 2}$ is equal to the laser frequency, $\omega=2 \pi \mathrm{c} / \lambda$. The critical density may be written as, $\mathrm{N}_{\mathrm{c}}=\left(\pi \mathrm{m}_{\mathrm{e}} \mathrm{c}^{2} / \mathrm{e}^{2} \lambda^{2}\right)$. At a laser wavelength of $1064 \mathrm{~nm}, \mathrm{~N}_{\mathrm{c}}=0.98 \times 10^{21} \mathrm{~cm}^{-3}$. Note that the ratio $\mathrm{N}_{\mathrm{e}} / \mathrm{N}_{\mathrm{c}}=\omega_{\mathrm{pe}}{ }^{2} / \omega^{2}$. The critical density plays an extremely important role in the interaction of electromagnetic waves with plasmas. This can best be illustrated by considering the dielectric function of the plasma, 


$$
\varepsilon(\omega)=1-N_{e} \frac{<\sigma>}{\omega}\left(i+\omega \tau_{m}\right)=1-\frac{\omega_{p e^{2}}}{\omega\left(\omega+i v_{m}\right)}
$$

The dielectric function determines both the refractive index of the plasma and absorption of electromagnetic waves within the plasma. The transmission of an electromagnetic wave is given by the Fresnel relation,

$$
T=\left|\frac{2 \operatorname{Re} \sqrt{\varepsilon}(\omega)}{1+\sqrt{\varepsilon}(\omega)}\right|
$$

At the critical density, the dielectric function becomes, $\varepsilon_{\mathrm{cr}}(\omega)=1-1 /\left(1+\mathrm{iv} v_{\mathrm{m}} / \omega\right)$. Since $v / \omega<<1$ for all but the most collisional plasmas, $\varepsilon_{\mathrm{cr}}(\omega) \approx 0$, and $\mathrm{T} \approx 0$. Hence, once the electron density reaches the critical value, very little electromagnetic energy is transmitted.

Formation of a critical density plasma has an important effect in materials processing with short pulse lasers. Once the critical surface is formed, laser energy is either absorbed at that surface or reflected from it. For most plasmas, there is strong absorption at the critical surface since the scale length, $L$, is equal to or greater than the wavelength of the incident light, $\mathrm{L}>\lambda .{ }^{20}$ Now, the maximum scale length is $\mathrm{L}_{\max }=\mathrm{v}_{\mathrm{s}} \tau_{\mathrm{p}}$ where the ion sound velocity, $\mathrm{v}_{\mathrm{s}}=\left(\mathrm{ZkT}_{\mathrm{e}} / \mathrm{m}_{\mathrm{ion}}\right)^{1 / 2} \approx 6 \times 10^{5} \mathrm{~cm} / \mathrm{sec}$. Since the pulses of interest here are $<10 \mathrm{ps}$, the maximum scale length is $\mathrm{L}_{\max } \approx 0.1 \mu \mathrm{m}$, which is much less than the incident wavelength. In the limit that $L<<\lambda$, electromagnetic waves are reflected from the critical surface and not absorbed. In Figure 5, we show the reflectivity of a normally transparent fused silica surface as a function of incident laser intensity. Near the damage threshold $\left(\approx 10^{13} \mathrm{~W} / \mathrm{cm}^{2}\right)$, critical density is not produced until late in the pulse (Figure 4). Only the last part of the laser pulse will experience any strong reflection. However, by operating high above threshold, critical density is achieved early in the pulse and reflectivities exceeding $90 \%$ can be obtained. By measuring the phasefront of the reflected waves, the researchers showed that the maximum scalelength in the plasma was $<0.1 \lambda .{ }^{21}$

The damage threshold of fused silica calculated according to the plasma model just described is compared to measured damage thresholds at both $526 \mathrm{~nm}$ and $1053 \mathrm{~nm}$ from $10 \mathrm{fs}$ to over $10 \mathrm{ps}$ in Figure 6. The theoretical damage fluence defining damage as the point at which the free electron density reaches the plasma critical density $\left(\approx 10^{21}\right.$ $\mathrm{cm}^{-3}$ ) is calculated with no adjustable parameters (Fig. 6, solid lines). Due to the rapid avalanche following production of the seed electrons by multiphoton ionization, the predicted damage threshold is only weakly dependent upon the actual free electron density at which damage occurs. A lower limit would correspond to the condition that the energy density of the conduction electrons equals the binding energy of the lattice $\left(\approx 10^{19} \mathrm{~cm}^{-3}\right)$. Use of this lower limit serves only to reduce the predicted damage threshold by $\approx 20 \%$. In addition to the good agreement with the measurements 


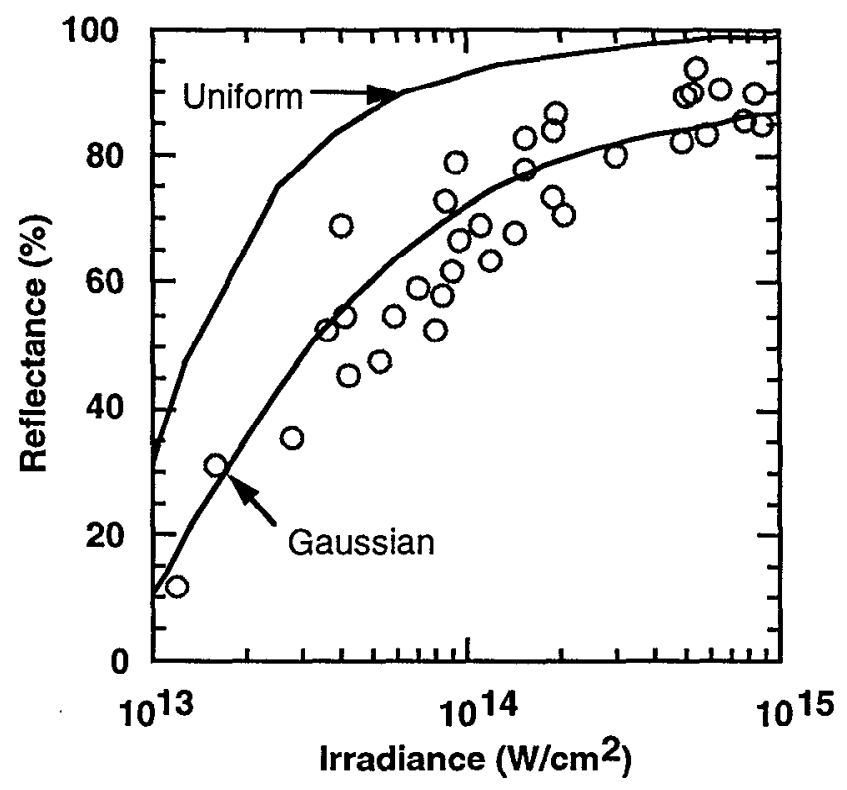

Figure 5. Reflectivity of fused silica surface as a function of laser irradiance for $\lambda=1053 \mathrm{~nm}$ and $\tau_{\mathrm{p}}=350 \mathrm{fs}$. Calculated reflectivities using the model described in the text for a gaussian and uniform beam are shown for comparision.

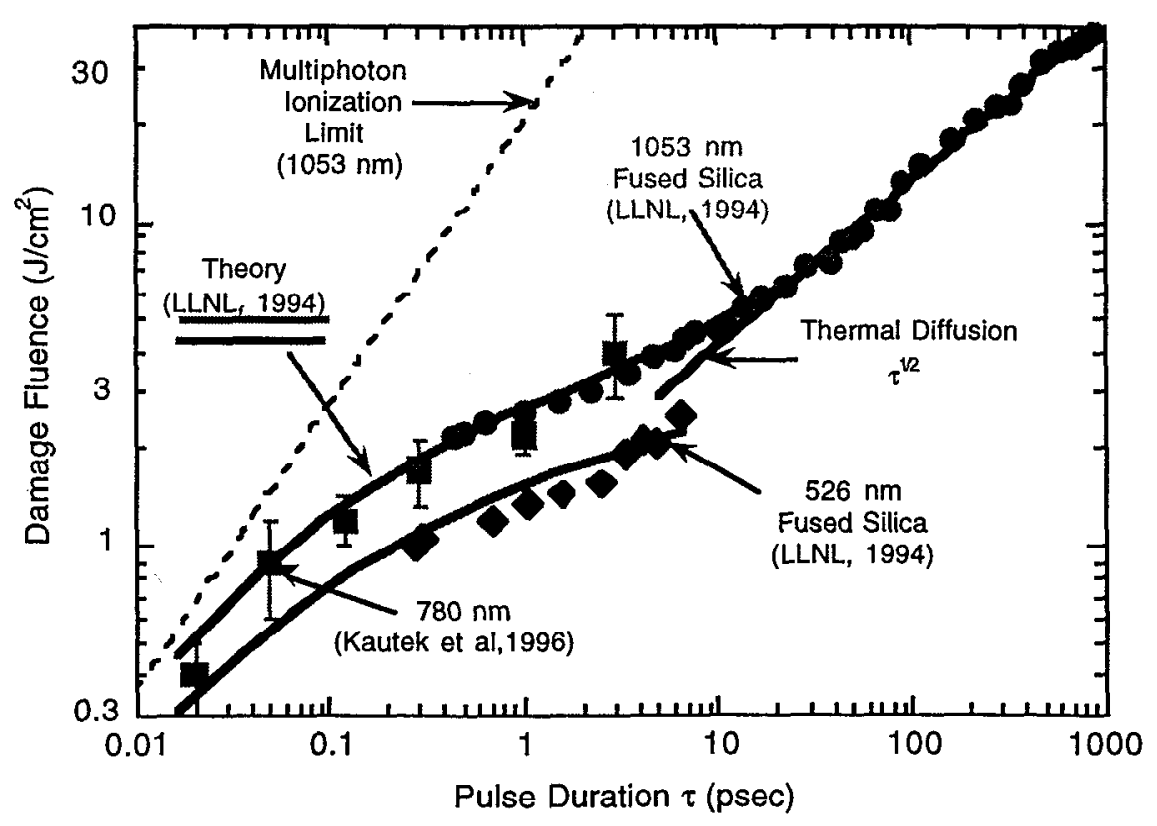

Figure 6. Measured and calculated damage thresholds of fused silica in the infrared and visible $(526 \mathrm{~nm})$. Deviation from $\tau^{1 / 2}$ scaling below $\approx 10 \mathrm{ps}$ indicates the transition to an ionization dominated damage mechanism. The calculated threshold for ablation by multiphoton ionization alone is shown as the dashed line. Data is taken from refs. 9 and 26. 
presented here, experiments by Auderbert, ${ }^{22}$ von der Linde, ${ }^{23}$ Kautek, ${ }^{24}$ and Kraucsz, ${ }^{25}$ are also in good agreement with our calculations but not those of $\mathrm{Du}^{26}$. For pulses less than $100 \mathrm{fs}$, the predicted damage threshold asymptotically approaches the multiphoton limit. In the long-pulse regime ( $\tau>20 \mathrm{ps}$ ), the data fit well to a $\tau^{1 / 2}$ dependence, characteristic of transfer of electron kinetic energy to the lattice and diffusion during the pulse. The damage is thermal in nature and characterized by melting and boiling of the surface. For long pulses, heating of the lattice and subsequent thermal damage can occur without significant collisional ionization. ${ }^{13}$

A significant feature of this non-thermal material removal mechanism is that since there is minimal energy deposition in the remaining material, there is a minimal increase in temperature. Thermal measurements show that when irradiated with conventional nanosecond laser pulses, the bulk temperature of a 1-mm slice of tooth increased by over $40^{\circ} \mathrm{C}$ while for femtosecond pulses the temperature rise was less than $2^{\circ} \mathrm{C}$ (Figure 7). The fluence in each case was set to remove approximately $1-\mu \mathrm{m}$ depth of material per pulse. This required $30 \mathrm{~J} / \mathrm{cm}^{2}$ for the nsec pulses and only $3 \mathrm{~J} / \mathrm{cm}^{2}$ for the femtosecond pulses. The practical consequences in dentistry are substantial. In the case of existing laser systems, active cooling of the tooth is necessary to prevent permanent damage to the pulp and nerves, which occurs at an increase of $\approx 5^{\circ} \mathrm{C}$ over body temperature. With ultrashort laser pulses, no cooling would be necessary.

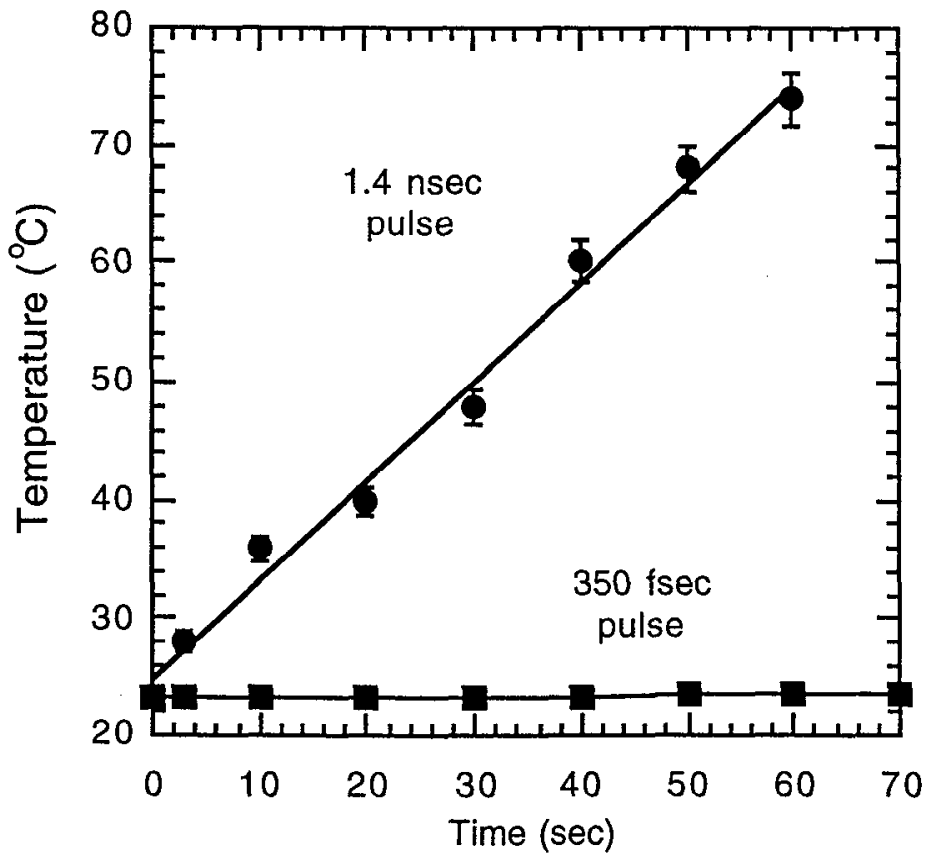

Figure 7. Temperature increase of bulk tooth due to drilling with 1.4-nanosecond (circles) and 350-femtosecond (squares) laser pulses. In both cases, the laser wavelength was $1053 \mathrm{~nm}$, and the spot size was $300 \mu \mathrm{m}$. The fluence was adjusted to. achieve a material removal rate of $1 \mu \mathrm{m} /$ pulse at $10 \mathrm{~Hz}$ for both pulse durations. 
Another dramatic example is the machining of explosives with ultrashort laser pulses. Cutting and machining operations on energetic materials present significant safety challenges. With conventional machine tools, improper fixturing of the work, improper tool configuration, and improper cutting speeds have resulted in violent reactions during machining operations. Conventional laser pulses are often used to ignite explosives. However, with femtosecond pulses, plasma formation and material removal occur too fast for significant energy transfer to the remaining material.

Furthermore, since only a small amount of material is removed per laser pulse $(\approx 3 \mu \mathrm{m})$, there is negligible shock imparted to the remaining material. The shock wave that does exist decays to an insignificant level within $\approx 1 \mu \mathrm{m}$ of the surface. The waste products from short-pulse laser cutting are, for the most part, solid carbon or benign gases, which can be released into the atmosphere.

We have machined a variety of high explosives including LX-14 (95.5\% HMX/4.5\% Estane), LX-15 (95\% HNS/5\% Kel-F), LX-16 (96\%PETN/4\%FPC 461), LX-17 (92.5\% TATB $/ 7.5 \%$ Kel-F), PBX-9407 (94\% RDX/6\% Exon 461), and pressed TNT. In some of the experiments the beam first cut through the HE and then into a stainless steel substrate and in other experiments the beam first cut through stainless steel and then into the HE. Figure 8 shows two cuts across a 1-cm-diameter, 2-mm-thick pellet made using a 1-kHz, 100-fs Ti:sapphire laser system. Fourier Transform Infrared Spectroscopy of the laser cut LX-16 surface showed no evidence of any chemical reaction products. The laser cut surface was chemically identical to the original LX-16 material. We also observed very high aspect ratio cuts and holes $(\approx 1000: 1)$ in high explosives. When we used pulses only modestly in the conventional regime (600 ps) deflagration of the LX-16 pellet was immediately observed (Figure $8 b$ ). Examination of the pellet afterward revealed that the edges of the cut were melted and contained a multitude of reaction products consistent with thermally induced ignition.
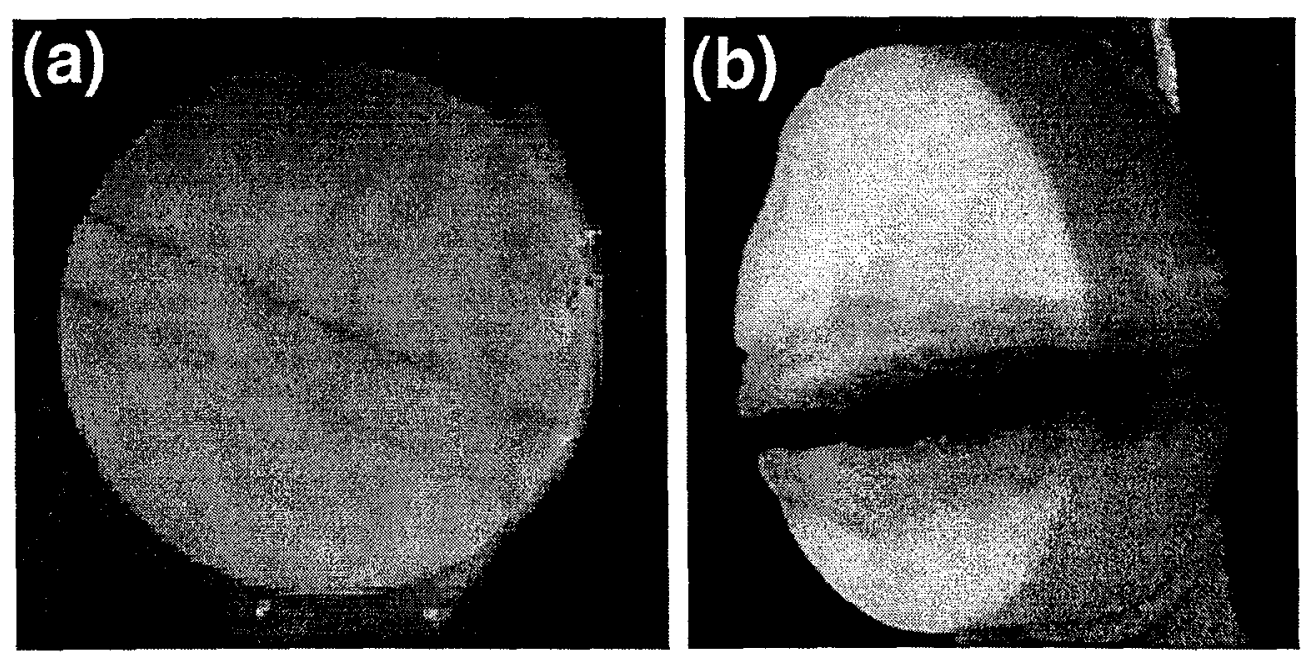

Figure 8. Cuts in explosive pellet (PETN) by a Ti:sapphire laser operating at $120 \mathrm{fs}$ (a) and $0.6 \mathrm{~ns}(\mathrm{~b})$. Thermal deposition in the long-pulse case caused the pellet to ignite. 
In summary for dielectric materials, there is a strong deviation from the usual $\tau^{1 / 2}$ scaling of laser damage fluence for pulses below $10 \mathrm{ps}$. The damage threshold continues to decrease with decreasing pulsewidth, but at a rate slower than $\tau^{1 / 2}$ in the range 0.1 to $10 \mathrm{ps}$. As the pulses become shorter than $\approx 0.1 \mathrm{ps}$, the increasing influence of multiphoton ionization results in a rapid decrease of the damage threshold. Multiphoton ionization provides an upper limit to the damage fluence for short pulses, preventing any increase in damage threshold with decreasing pulse duration as might be predicted from a pure avalanche model. This new mechanism of damage (material removal) is accompanied by a qualitative change in the morphology of the interaction site. The damage site is limited to only a small region where the laser intensity is sufficient to produce a plasma with essentially no collateral damage. This process enables high-precision machining of all dielectrics with no thermal shock or distortion of the remaining material. Although the absolute damage fluence varies, all pure dielectrics (oxides, fluorides, explosives, plastics, glasses, ceramics, etc.) exhibit similar behavior. All dielectrics share the same general properties of slow thermal diffusion and an electron-phonon scattering rate slower than the rate at which energy is absorbed from the field by the free electrons. All dielectrics should therefore exhibit similar shortpulse machining characteristics.

\section{Metals}

Metals are machined either with abrasive techniques (lathes, saws, drills), or by localized thermal processing (conventional lasers, electron beam, plasma or acetylene torch, etc.). The basic interaction in localized thermal processing is the deposition of energy from the incident beam in the material of interest in the form of heat (lattice vibrations). In the case of the laser, the absorption of energy is dependent upon the optical properties of the metal. The laser energy that is absorbed results in a temperature increase at and near the absorption site. The magnitude and spatial extent of the temperature achieved during the laser pulse is strongly dependent upon the thermomechanical properties of the metal. As the temperature increases to the melting or boiling point, material is removed by conventional melting or vaporization. ${ }^{27}$ Depending on the pulse duration of the laser, the temperature rise in the irradiated zone may be very fast, resulting in thermal ablation and shock. The irradiated zone may be vaporized or simply ablate off due to the fact that the local thermal stress has become larger than the yield strength (thermal shock). Plasma formation may even result in the vaporized plume. In all these cases, there is an impact on the material surrounding the site where material has been removed. The surrounding material will have experienced a large temperature excursion or shock often resulting in significant change to the material properties. These changes may range from a change in grain structure to an actual change in composition. Such compositional changes include oxidation (if cut in air) or, in the case of alloys, changes in composition of the alloy. This heat affected zone may range from a few microns to millimeters depending on the thermomechanical properties of the metal, laser pulse duration and other factors (e.g., active cooling). In 
many applications, the presence of the heat or shock effected zone may be severely limiting since the material properties of this zone may be quite different than that of the bulk. Furthermore, small-scale devices (features on the order of a few tens of microns) cannot tolerate the thermal stress induced in the material during the machining process.

Another limitation of conventional laser or electron beam processing in certain applications is the lack of precision and the presence of redeposited or resolidified material. As mentioned previously, cutting or drilling occurs by either melting or vaporizing the material of interest. The surface adjacent to the removed area will have experienced significant thermal loading, often resulting in melting. This melting can be accompanied by flow prior to solidification. This can result in the deposition of slag surrounding the kerf (Figure 9a) which, in many high-precision applications, is unacceptable. Also, the resolidification process is not uniform, resulting in a lack of precision in the machined surface. In the cases where the deposition of conventional slag can be prevented, redeposition of vaporized material on the walls or upper surface of the kerf is common. This condensate often reduces the quality of the cut and decreases the cutting efficiency since the beam must again remove this condensate
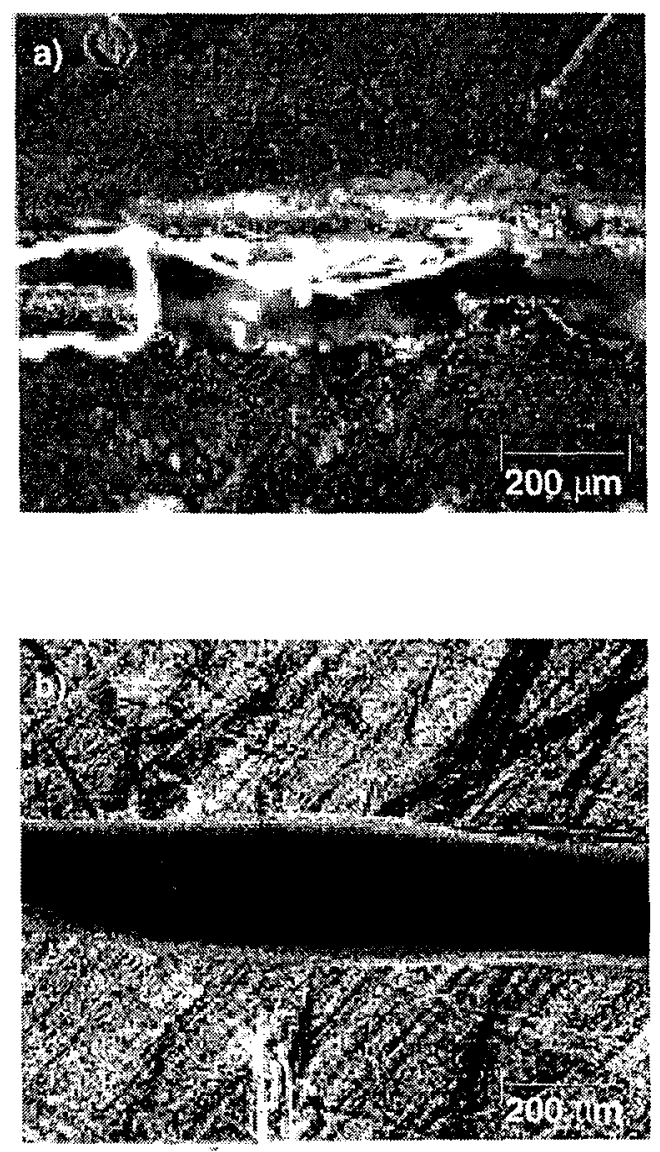

Figure 9. Cuts in stainless steel performed with 1054-nm laser pulses with a duration of (a) $1.4 \mathrm{~ns}$ at a fluence of $52 \mathrm{~J} / \mathrm{cm}^{2}$ and (b) $350 \mathrm{fs}$ and $14 \mathrm{~J} / \mathrm{cm}^{2}$. The fluence was adjusted to achieve a material removal rate of $0.5 \mu \mathrm{m} /$ pulse in both cases. 
before interacting with the bulk material underneath. Many of these limitations can be reduced by the use of secondary techniques to aid the cutting process. The most common of these are active cooling of the material of interest either during or immediately following the laser pulse, and the use of high pressure gas jets to remove vaporized or molten material from the vicinity of the cut. These techniques can be effective at improving the kerf at the cost of an increase in system complexity and often a decrease in cutting efficiency.

As in the case of dielectrics, ultrashort pulses enable the laser cutting/machining of metals and alloys with high machining speed, extreme precision, negligible heat affected zone, and no modification to the material surrounding the kerf. Unlike dielectrics however, there is no multiphoton ionization step necessary since the metal already contains "free" electrons in the conduction band. These electrons will absorb the laser light via inverse Bremstrahlung heating (collisional absorption) as described previously. Again, a Fokker-Planck equation can be established to describe the energy transfer from the electrons to the lattice (electron-phonon coupling) and heat transfer directly by electron conduction away from the absorption site. Historically, this situation has been described by a simple two temperature model for the ion and electron temperatures. ${ }^{28}$ Since on the time scales of interest, the temperature gradients in transverse dimensions are negligible relative to that in the longitudinal dimension, a one-dimensional description is adequate:

$$
\mathrm{C}_{\mathrm{e}} \frac{\partial \mathrm{T}_{\mathrm{e}}}{\partial \mathrm{t}}=\frac{\partial}{\partial \mathrm{z}}\left(\mathrm{k} \frac{\partial \mathrm{T}_{\mathrm{e}}}{\partial \mathrm{z}}\right)-\gamma\left(\mathrm{T}_{\mathrm{e}}-\mathrm{T}_{\mathrm{i}}\right)+\mathrm{A}(\mathrm{z}, \mathrm{t})
$$

and

$$
\mathrm{C}_{\mathrm{i}} \frac{\partial \mathrm{T}_{\mathrm{i}}}{\partial \mathrm{t}}=\gamma\left(\mathrm{T}_{\mathrm{e}}-\mathrm{Ti}\right)
$$

where $C_{e}$ and $C_{i}$ are the heat capacity of the electrons and ions (lattice) respectively, $\gamma$ is the rate that electron energy is dissipated to the lattice via phonons $\left(\mathrm{W} / \mathrm{cm}^{3}-\mathrm{o} \mathrm{K}\right)$, and $\mathrm{A}(\mathrm{z}, \mathrm{t})$ is the source term. Since the conductivity of the lattice is much lower than the electron-phonon transfer rate, $\mathrm{k}_{\mathrm{i}}<<\gamma$, the term representing conduction through the lattice has been neglected in (eq. 15). Since the time scale for energy transfer from the electrons to the lattice via phonon scattering is finite, short laser pulses can heat the electrons to high temperatures with very little energy transfer to the ions (lattice). Hence, the need for a two temperature description. For a simple metal, the electron heat capacity and conductivity are described by the Fermi relations,

$$
\mathrm{C}_{\mathrm{e}} \approx \frac{\pi^{2}}{2}\left(\frac{k \mathrm{~T}_{\mathrm{e}}}{E_{F}}\right) \mathrm{N}_{\mathrm{e}} k \text { and } \mathrm{k}_{\mathrm{e}}=\frac{2}{3} \frac{E_{F}}{\mathrm{~m}_{\mathrm{e}} v} \mathrm{C}_{\mathrm{e}}
$$

where $E_{F}$ is the Fermi energy $(\approx 7 \mathrm{eV})$ and $v=v_{\mathrm{ee}}+v_{\mathrm{ei}}$ is the sum of the electron-electron collision frequency $v_{\mathrm{ee}}$ and the electron-lattice (ion) collision frequency, $v_{\mathrm{ei}}$. In the limit that $\gamma \rightarrow \infty, T_{e}=T_{i}=>T$ and eq. 14 reduces to the usual one-dimensional heat conduction 
equation for the temperature, T. Simplified versions of these eqs. 14, 15 have been solved analytically and provide good insight into the important physical processes. ${ }^{29}$

A solution of these equations in the limit of fast equilibration between the ions and electrons for gold irradiated at $1053 \mathrm{~nm}$ and $0.5 \mathrm{~J} / \mathrm{cm}^{2}$ is given in Figure 10 . The thermal penetration depth achieved during the pulse, $L_{t h}=2 \sqrt{ } \alpha \tau$, is less than one micron resulting in a very small amount of material ( $0.01-1$ micron) heated to above the boiling point with extremely small transport of energy either by shock or thermal conduction away from the volume of interest. For pulses with a duration below the characteristic energy transfer time between electrons and the lattice, the model described by eqs. 14 and 15 predicts a damage threshold which no longer scales as $\tau^{1 / 2}$ as is predicted by a conventional thermal mechanism of damage. Instead, the damage threshold is predicted to be essentially independent of pulse duration. ${ }^{30}$ This behavior is observed in all metals which we have investigated. In Figure 11, we show the damage threshold of gold measured at $1053 \mathrm{~nm} .{ }^{31}$ The essential constancy of the damage threshold below $\approx 80 \mathrm{ps}$ is readily observed and the data is well fit by the two temperature model with $\gamma=3.5 \times 10^{11} \mathrm{~W} / \mathrm{cm}^{3}-{ }^{\circ} \mathrm{K}$.

For real machining applications, the surface of the metal is heated far beyond its boiling point and the simple two temperature model breaks down. Instead, a full radiative hydrodynamic model is required that accounts for plasma formation, multiple ionization, material equation of state and shock wave generation and cooling by radiation, conduction and plasma expansion ( $\mathrm{PdV}$ work). A one-dimensional EulerLagrangian code was developed for this purpose. ${ }^{32}$ The predicted pressure and temperature of aluminum irradiated in the ultrashort-pulse regime ( $350 \mathrm{fs}$ ) and the conventional (ns) regime are given in Figures 12-14. For the femtosecond

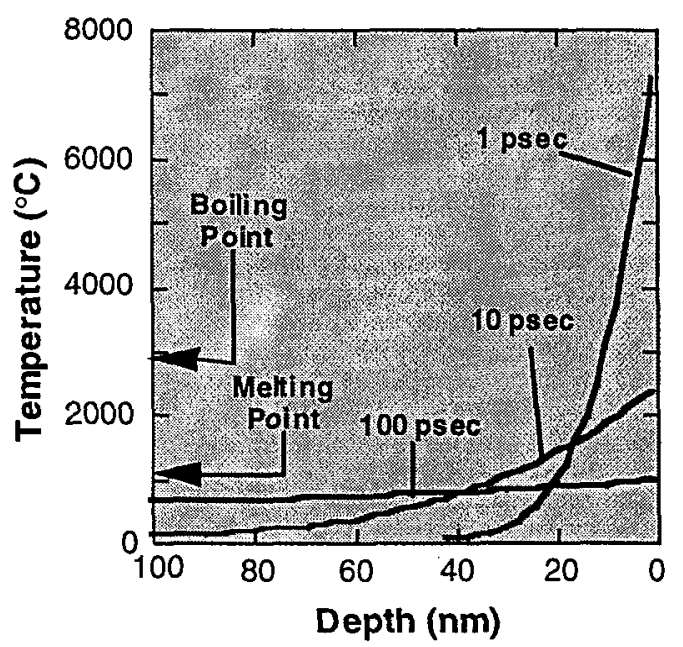

Figure 10. Calculated temperature profiles in gold irradiated at $1054 \mathrm{~nm}$ and a fluence of $0.5 \mathrm{~J} / \mathrm{cm}^{2}$ for 1,10 , and 100 ps pulses. 


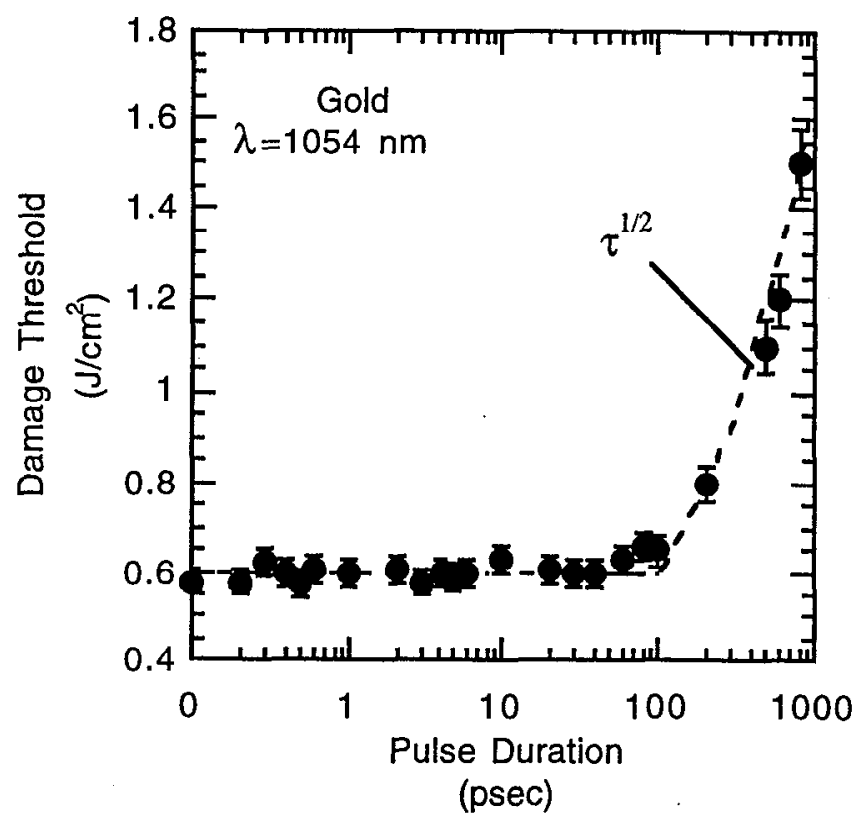

Figure 11. Damage threshold of gold at $1054 \mathrm{~nm}$ as a function of pulse duration [from ref. 29]

pulse focused to $5 \mathrm{~J} / \mathrm{cm}^{2}\left(1.4 \times 10^{13} \mathrm{~W} / \mathrm{cm}^{2}\right)$, the absorbed laser energy heats the skin depth of the material to $\approx 12.5 \mathrm{eV}$ (Figure $12 \mathrm{~b}$ ). In the next few picoseconds, this energy is dissipated into the bulk by both shock and electron conduction and is also dissipated by the initial plasma expansion off the surface and radiation. A depth of $\approx 0.1$ micron is heated to near $1 \mathrm{eV}$. This material blows off the surface with a static expansion velocity of $2 \times 10^{6} \mathrm{~cm} / \mathrm{s}$ over the next nanosecond (Figure 13b). Note that the solid left behind remains essentially at room temperature even within $\approx 1 \mu \mathrm{m}$ of the machined surface. The initial shock wave launched into the material is large reaching $2 \mathrm{Mbar}$. This shock propagates with a velocity $=6 \times 10^{5} \mathrm{~cm} / \mathrm{s}$ but dissipates rapidly in magnitude. The pressure associated with the shock drops to less than $100 \mathrm{kbar}$ (less than the yield strength of the material) within the first micron (Figure 13). This offers extremely highprecision machining with no heat or shock effected zone extending beyond $\approx 1 \mu \mathrm{m}$ of the machined surface. In the case of a 1-ns pulse, the majority of the laser energy is absorbed in the expanding plasma. The energy that strikes the surface heats a depth of $\approx 2 \mu \mathrm{m}$ to the boiling point and a few microns to the melting point (Figure 14). For the even longer pulses more typical of laser machining, the temperatures achieved are below the ionization threshold and standard thermal models (which only account for melting and boiling) are adequate.

The lack of significant energy deposition beyond the volume of interest achieved by using ultrashort pulses enables the use of high repetition $(0.1-100 \mathrm{kHz})$ lasers without the need for external cooling of the part being machined. Even though only a very small depth of material is removed per pulse, the high repetition rate enables extremely high cut rates (beyond $1 \mathrm{~mm}$ depth per second). Another feature of the very short pulse 
duration is the fact that there is no vaporization or transport of material during the pulse (Figure 12). During the pulse, there is insufficient time for hydrodynamic expansion of the vaporized material. As a result, the laser encounters the solid surface for the duration of the pulse depositing energy into solid density material.
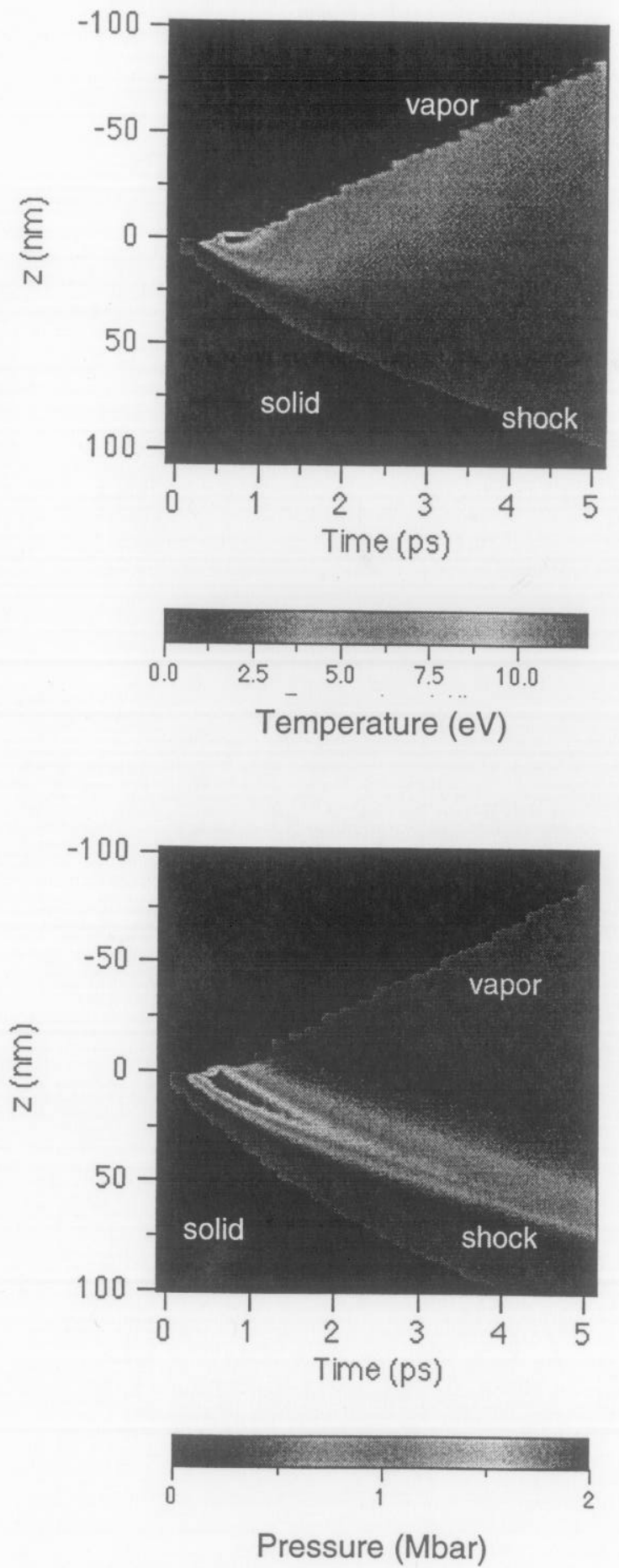

Figure 12. Calculated temperature (a) and pressure (b) of aluminum irradiated by 1053-nm, 350-fs laser pulses at a fluence of $5 \mathrm{~J} / \mathrm{cm}^{2}$. 

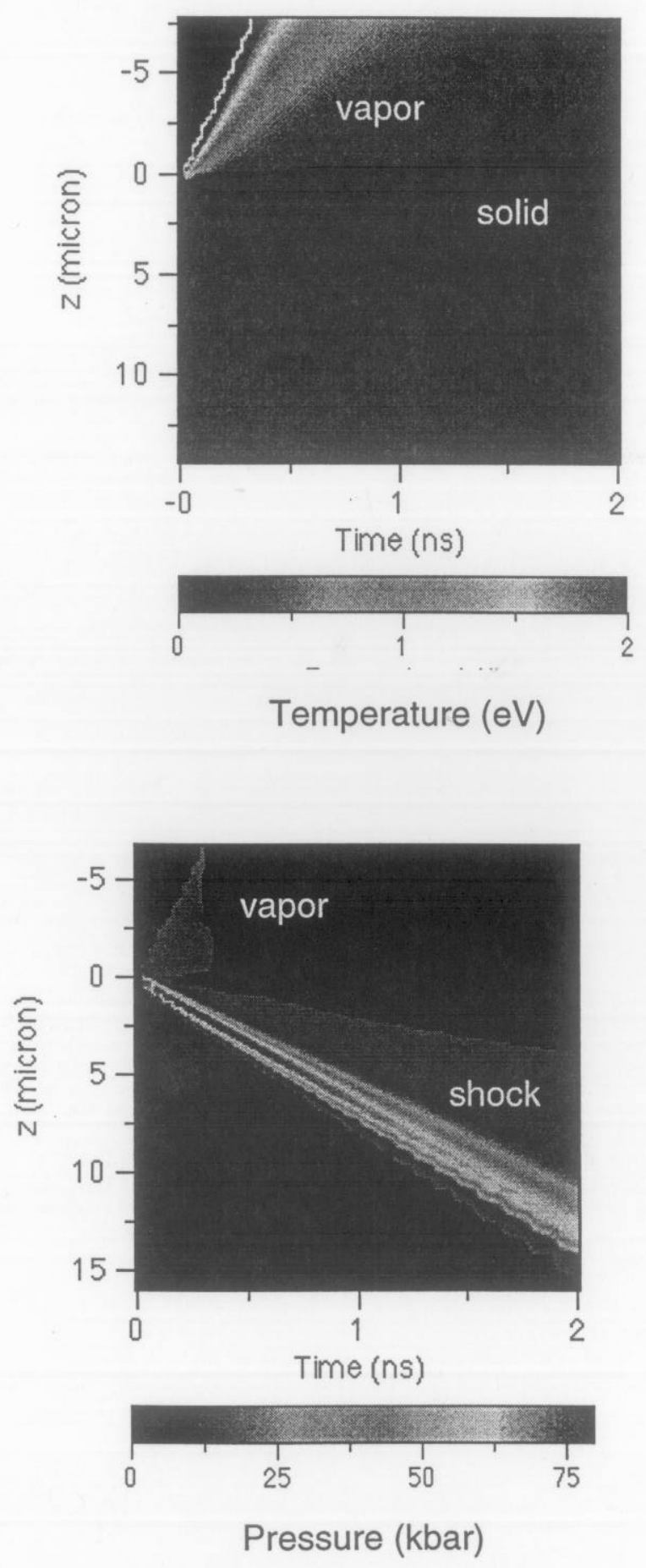

Figure 13. Calculated temperature (a) and pressure (b) of aluminum following irradiation by $1053-\mathrm{nm}, 350-\mathrm{fs}$ laser pulses at a fluence of $5 \mathrm{~J} / \mathrm{cm}^{2}$. The calculation is carried out to two nanoseconds showing plasma blowoff and decay of the shock wave. 

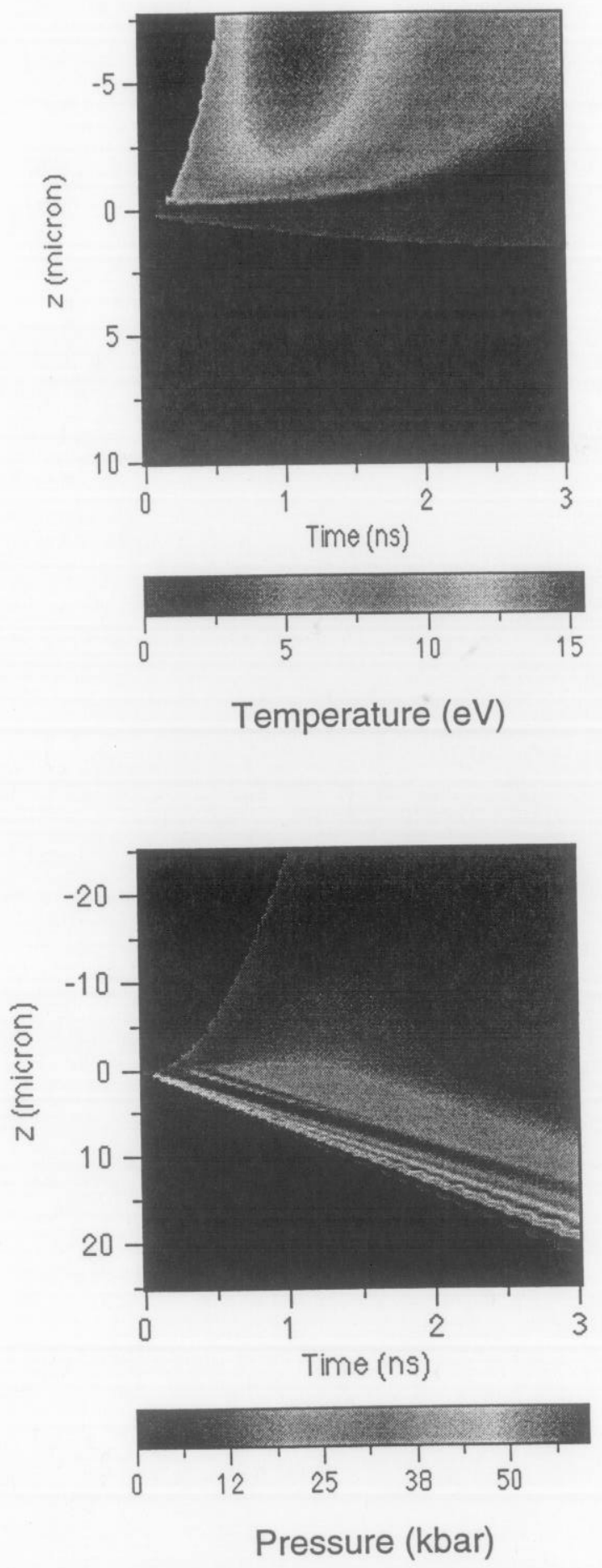

Figure 14. Calculated temperature (a) and pressure (b) of aluminum following irradiation by a 1053-nm, 1-ns laser pulse at a fluence of $20 \mathrm{~J} / \mathrm{cm}^{2}$ (same depth of material removed as in Figure 13). 
While the typical temperatures achieved at the damage threshold may be only a fraction of an electron volt $\left(1000-3000^{\circ} \mathrm{K}\right)$, those achieved for useful machining are between 1 and $100 \mathrm{eV}$. The temperature is determined by the product of the incident laser irradiance, $I\left(W / \mathrm{cm}^{2}\right)$, the square of the laser wavelength, $\lambda^{2}(\mu \mathrm{m})$, and the absorption characteristics of the metal. The difference between the threshold and the fluence required for useful machining is illustrated for stainless steel in Figure 15. At threshold, only a very small amount of material $(<0.1 \mu \mathrm{m})$ is removed per laser pulse. As the laser fluence is increased, the depth of material removed increases rapidly and then saturates at $\approx 12 \mathrm{~J} / \mathrm{cm}^{2}$. For the 120 -fs pulses used in these experiments, the peak irradiance was over $10^{14} \mathrm{~W} / \mathrm{cm}^{2}$ and produced an initial plasma temperature near $20 \mathrm{eV}$.

The high plasma temperatures associated with saturated material removal ensure that the vaporized material will be completely removed from the kerf without redeposition on the walls. For example, an expanding vapor with even a low expansion velocity of $10^{5} \mathrm{~cm} / \mathrm{sec}$ will be 1 meter away from the surface before the arrival of the next pulse $1 \mathrm{~ms}$ later (operating at $1 \mathrm{kHz}$ ). With conventional nanosecond or microsecond lasers, the vapor will evolve during the laser pulse (Figure 14). This reduces the coupling of the laser light to the solid surface since the incident laser light will be scattered and absorbed by the vapor.

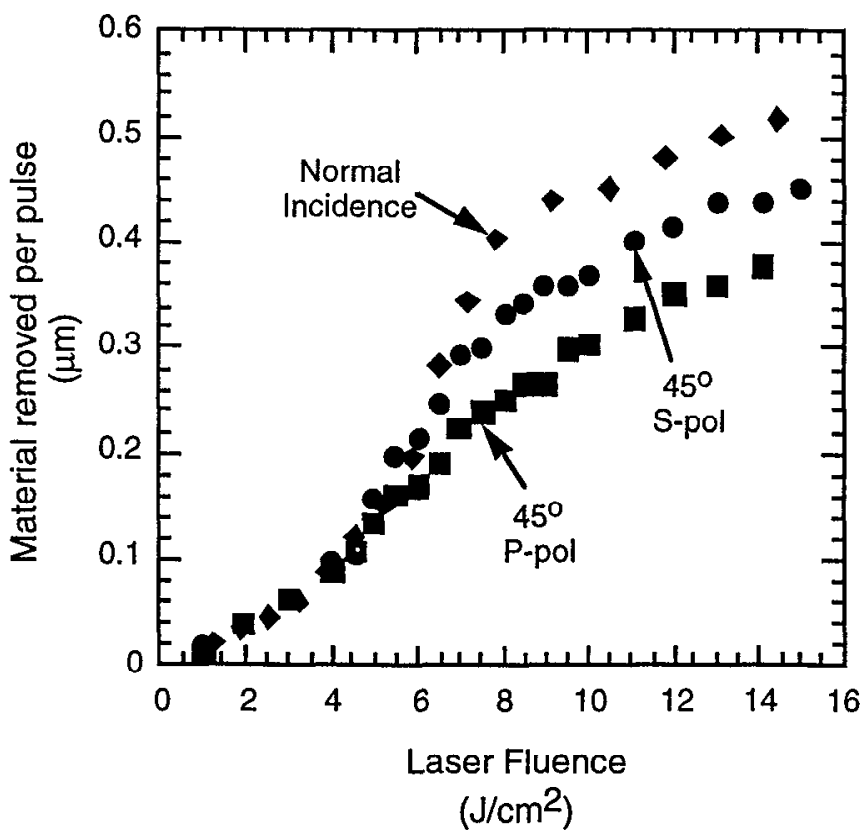

Figure 15. Depth of material removed per laser pulse for stainless steel and $\lambda=825 \mathrm{~nm}$. 
The cross-section of a $400-\mu \mathrm{m}$ hole in 0.8 -mm-thick stainless steel drilled at $45^{\circ}$ with 120-fs pulses shows that metallic grain structure is unaltered up to the edge of the hole (Figure 16). High resolution microscopy indicates that there is no heat or shock affected zone extending beyond $\approx 1 \mu \mathrm{m}$ away from the surface. The top (entrance) and bottom of the hole are typical of holes drilled with pulses having a Gaussian spatial profile. The shape of the hole is deterministic with no evidence of slag or heat-affected zone. Material is removed only in those regions where the beam intensity is sufficient to form a plasma. This deterministic nature of material removal makes possible the production of shaped cuts, holes or textured surfaces by shaping the spatial profile of the laser beam. Several researchers have used diffractive optics to modify the spatial profile of the beam to produce holes, with very steep side walls. ${ }^{33,34}$ A shaped fuel injector nozzle produced with 120 -fs pulses and a diffractive phase plate to produce a nearly flat top, $0.2-\mathrm{mm}$-diameter beam is shown in Figure 17.

High-aspect-ratio holes and cuts $(0.05-\mathrm{mm}$ diameter thru $1.0 \mathrm{~mm})$ have been produced in numerous metals and dielectrics. Production of high-aspect-ratio features requires consideration of the waveguide nature of the feature and associated beam shaping. Tailoring the beam to avoid possible condensation of the plasma plume on the walls must also be considered for the production of high aspect $(>8: 1)$ ratio features. Analysis of these issues is beyond the scope of this paper.

\section{Laser Technology}

The depth of material removed at the optimum fluence with ultrashort-pulse lasers is limited to $\approx 0.5 \mu \mathrm{m}$ per laser pulse for both dielectrics and metals. Hence, the rate of material removal scales essentially linearly with the repetition rate of the laser system. Since the optimum fluence is fixed by the material to be machined and the aspect ratio of the feature of interest, the area which can be machined per laser pulse is set by the energy of the pulses. Combining these features, the volumetric machining rate scales linearly with the average power of the laser. Ultrashort-pulse lasers are based on the concept of chirped-pulse amplification described for lasers by Fisher and Bischel. ${ }^{35}$ This technique was first realized to produce picosecond pulses in a solid-state laser system by Strickland and Mourou. ${ }^{36,37}$ Since the first demonstration, dramatic improvements in laser architecture, laser materials and technology have led to compact systems producing terawatt and even petawatt pulses. 38,39 These relatively high pulse energy systems are of limited use for machining applications. Instead, high repetition rate systems with only modest pulse energy $1-20 \mathrm{~mJ}$ are required. These systems are almost all based on Titanium-doped sapphire as the laser material. Commercial systems designed for scientific research are available at the $1-W$ level $(1 \mathrm{~mJ}$ per pulse at $1 \mathrm{kHz}$ repetition rate) and $2-W$ custom systems have been produced.

The first true machining system for use in industrial production was recently developed at Lawrence Livermore National Laboratory (Figure 18). The system is based on Ti:sapphire as the laser material and produces 1.5-mJ pulses with a duration of $120-\mathrm{fs}_{\mathrm{s}}$ and diffraction-limited beam quality. In order to achieve high machining speed, the 

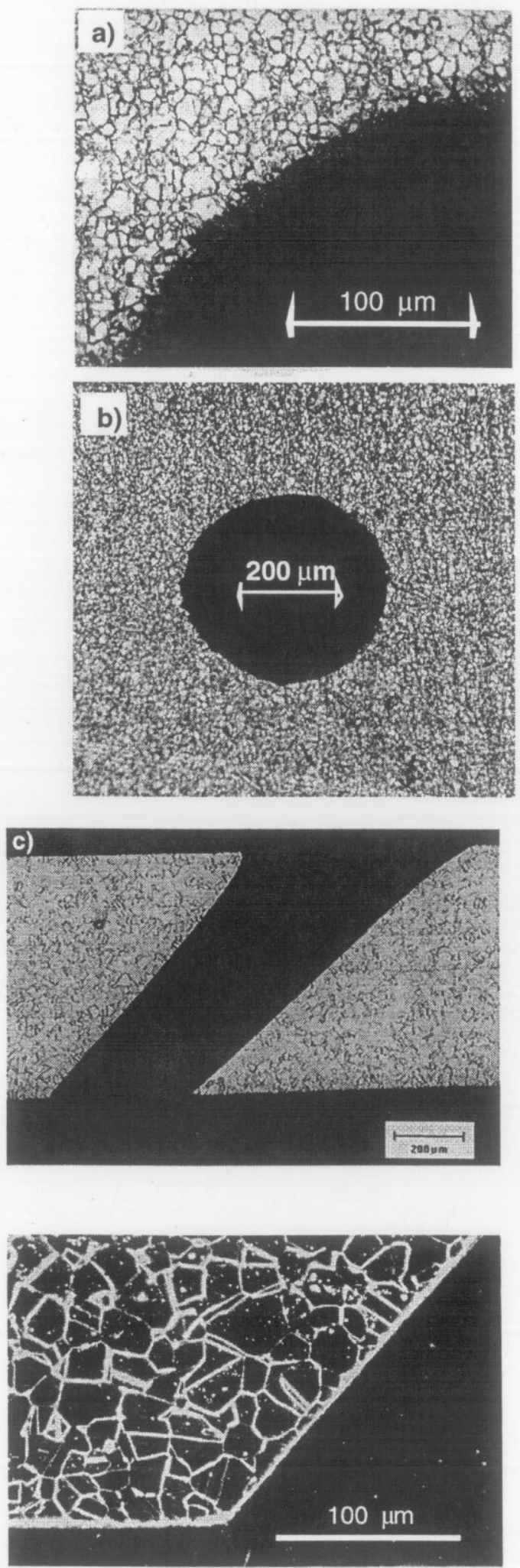

Figure 16. Holes drilled through $1 \mathrm{~mm}$ stainless steel with 120 -fs laser pulses at $1 \mathrm{kHz}$ at $45^{\circ}$. (a) Magnified section of top of hole, (b) exit hole on bottom, (c) cross section, (d) magnified (bottom left) section of cross section. 


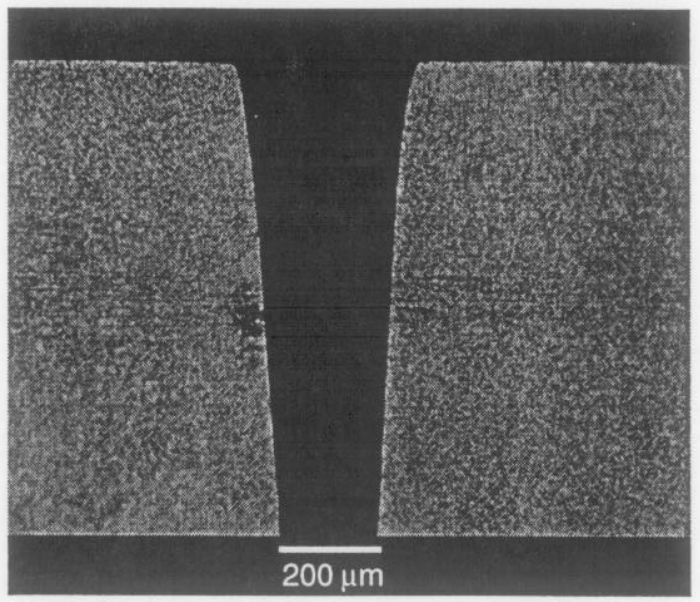

Figure 17. Holes drilled through $1 \mathrm{~mm}$ stainless steel for fuel injector nozzles.

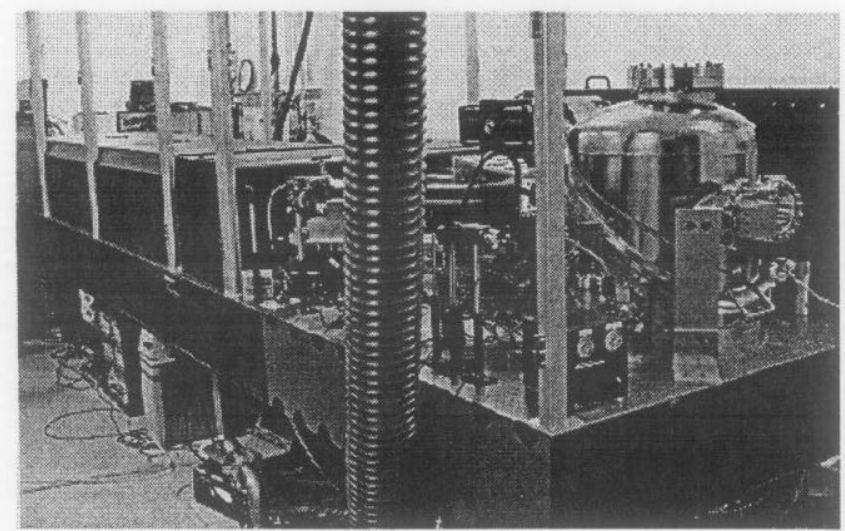

Figure 18. Photograph of a $15-\mathrm{W}$ average power femtosecond laser machining system developed for industrial operation.

repetition rate of the system is $10 \mathrm{kHz}$ resulting in an average power of $15 \mathrm{~W}$. The system is fully computer-controlled and is designed to be operated by a machinist. The system is modular in design and completely self-contained. Individual modules can be removed and replaced on the line to minimize any down time for maintenance.

Although Ti:Sapphire based ultrashort-pulse lasers can be scaled in average power up to $\approx 100 \mathrm{~W}$, the complexity of these systems will limit their use. This complexity arises from the short upper-state lifetime $(\tau \approx 2 \mu \mathrm{sec})$ of the laser material itself. The short energy storage time requires that the Ti:Sapphire be pumped by a second laser system. This laser-pumped laser requirement leads to a complex system where industrial quality standards of availability factor and ease of use will be difficult to achieve (particularly beyond the $\approx 20 \mathrm{~W}$ level). This complexity can be substantially reduced by the use of new laser materials which offer both broad-bandwidth (necessary to produce the short pulse) and a long upper-state lifetime enabling direct diode-pumping. Such systems offer the promise of kilowatt-class average power in a simplified, compact package. The first of these new direct diode-pumped systems is currently in development at LLNL. 


\section{Conclusion}

The use of ultrashort-pulse lasers allows materials processing of practically any material (diamond, $\mathrm{SiC}$, teeth, stainless steel, explosives, $\mathrm{TiC}$, etc.) with extremely high precision and minimal collateral damage. Advantages over conventional laser machining (using pulses longer than a few tens of picoseconds) are realized by depositing the laser energy into the electrons of the material on a time scale short compared to the transfer time of this energy to the bulk of the material (either by electron-phonon coupling or thermal diffusion). The process forms a critical density plasma, which then expands away from the surface following the laser pulse. High ablation efficiency is achieved with negligible energy deposition to the remaining material either by heat or shock. As a result, high-speed machining of materials to the micron scale can be realized. 


\section{Acknowledgements}

Ultrashort-pulse laser machining is now being developed at a variety of locations throughout the world. We would like to acknowledge other pioneering work in this field by P. Callen, E. Glenzer, W. Kautek, C. Momma, G. Mourou, and S. Nolte and thank them for many helpful discussions. We also thank P. Armstrong, N. Frank, B. Pyke, D. Shipley and others involved in the design and construction of the femtosecond machining system. This work was performed under the auspices of the U.S. Department of Energy by Lawrence Livermore National Laboratory under contract No. W-7405ENG-48. 


\section{References}

1. S. C. Jones, P. Braunlich, R. T. Casper, X.-A. Shen, and P. Kelly, Opt. Eng.28, 1039 (1989).

2. N. Bloembergen, IEEE J. Quantum Electron. QE-10, 375 (1974).

3. Laser Induced Damage in Optical Materials, Proceedings of the Boulder Damage Symposium: Vols. 1-25 (1969-93).

4. A.A. Manenkov and A.M. Prokhorov, Sov. Phys. Usp. 29, 107 (1986).

5. M. Sparks, D. L. Mills, R. Warren, T. Holstein, A. A. Maradudin, L. J. Sham, E. Loh Jr., and D. F. King, Phys. Rev. B 24, 3519 (1981).

6. R.M. Wood, Laser Damage in Optical Materials (Hilger, Boston, 1986).

7. J.H. Campbell, F. Rainer, M. Kozlowski, C.R. Wolfe, I. Thomas, and F. Milanovich, in Laser Induced Damage in Optical Materials, SPIE Vol. 1441, 444 (1990).

8. M.D. Perry and E.M.Campbell, Petawatt Laser Proposal, LLNL Internal Report (1992)

9. B.C. Stuart, M.D. Feit, A.M. Rubenchik, B.W. Shore, and M.D. Perry, Phys. Rev. Lett., 74, 2248 (1995).

10. D. Du, X. Liu, G. Korn, J. Squier and G. Mourou, Appl. Phys. Lett., 264, 917 (1994).

11. X.A. Shen, S.C. Jones, and P. Braunlich, Phys. Rev. Lett. 62, 2711 (1989).

12. L. H. Holway Jr. and D. W. Fradin, J. Appl. Phys. 46, 279 (1974).

13. D. Arnold, E. Cartier and D. J. DiMaria, Phys. Rev. A 45, 1477 (1992).

14. B. K. Ridley, Quantum Processes in Semiconductors (Clarendon, Oxford, 1993).

15. M. D. Perry and O. L. Landen, Physical Review A, 38, 2815(1988).

16. L.V. Keldysh, Sov. Phys., JETP 20, 1307 (1965).

17. M. D. Perry, A. Szoke, O. L. Landen, and E. M. Campbell, Phys. Rev. Lett. 60, 1270 (1988); ibid,Phys. Rev. A, 37, 747 (1988).

18. M.V. Ammosov, N.B. Delone, and V.P. Krainov, Sov. Phys., JETP, 64, 1191 (1986).

19. B.C. Stuart, M.D. Feit, S. Herman, A. Rubenchik, B.W. Shore and M.D. Perry, Phys. Rev. B, 53 (1996).

20. W.Kruer, Physics of Laser Plasma Interactions,

21. M.D. Perry, V. Yanovsky, A.M. Rubenchik and M.D. Feit; Plasma Mirrors, submitted to Physics of Plasmas, (1998).

22. P. Audebert, Ph. Daguzan, S. Guizard, K. Krastev, P. Martin, G. Petite, A. Dos Santos, and A. Antonetti, Phys. Rev. Lett., 73, 1990 (1994).

23. D. von der Linde and H, Schuler in Proceedings of Short Wavelength VI: Physics with High Intensity Pulses, St. Malo, France (1994).

24. W. Kautek, J. Kruger, M. Lenzner, S. Sartania, C. Spielmann, F. Krausz, Appl. Phys. Lett. 69, 3146 (1996).

25. F. Krausz, et al, Phys. Rev. Lett., to be published.

26. D. Du, X. Liu, J. Squier, and G. Mourou, Appl. Phys. Lett., 64, 3071, (1994).

27. J.F. Ready, Effects of High-Power Laser Radiation, Academic Press, New York, 1971.

28. S.I. Anisimov, B.L. Kapeliovich and T.L. Perlman, Sov. Phys. JETP, 39, 375 (1974).

29. S, Nolte, C. Momma, H. Jacobs, A. Tunnermann, B. Chichkov, B. Wellegehausen, . and H. Welling, J. Opt. Soc. Amer. B, 14, 2716 (1997). 
30. P.B. Corkum, F. Brunel, N.K. Sherman, and T. Srinivasan-Rao, Phys. Rev. Lett., 61, 2886 (1988).

31. M.D. Perry, et al, Conference on Lasers and Electrooptics, Baltimore May 1994;

B. Stuart, S. Herman, M.D. Feit, B.W. Shore, and M.D. Perry, J. Opt. Soc. Amer. B, 13, 459 (1996).

32. A.M. Rubenchik, M.D. Feit, M.D. Perry and J.T. Larsen, App. Surface Science, 127, 193 (1998).

33. M.D. Perry, B.C. Stuart, H. Nguyen, M.D. Feit, and S. Dixit, OSA Annual Meeting, Portland (1996).

34. S. Nolte, C. Momma, et al, OSA Annual Meeting, Long Beach, CA (1997)

35. R.A. Fisher and W. Bischel, "Pulse Compression for more efficient operation of solid-state laser amplifier chains, Appl. Phys. Lett., 24, 468 (1974); ibid,IEEE J. Quan. Elec., QE-11, 46 (1975).

36. D. Strickland and G. Mourou, Opt. Comm. 56, 219 (1985).

37. P. Maine, D. Strickland, P. Bado, M. Pessot, and G. Mourou, IEEE J. Quantum Electron. 24, 398 (1988).

38. M.D. Perry and G. Mourou, "Terawatt to Petawatt Lasers," Science 264, 917 (1994).

39. G. Mourou, C.P. Barty and M.D. Perry, Physics Today, p. 22, January (1998) 